Research Article

\title{
Vertex-Disjoint Paths in a 3-Ary $n$-Cube with Faulty Vertices
}

\author{
Xiaolei Ma and Shiying Wang $(D)$ \\ College of Mathematics and Information Science, Henan Normal University, Xinxiang, Henan 453007, China \\ Correspondence should be addressed to Shiying Wang; shiying@sxu.edu.cn
}

Received 14 May 2020; Revised 29 June 2020; Accepted 8 July 2020; Published 5 August 2020

Guest Editor: Carlos Llopis-Albert

Copyright (c) 2020 Xiaolei Ma and Shiying Wang. This is an open access article distributed under the Creative Commons Attribution License, which permits unrestricted use, distribution, and reproduction in any medium, provided the original work is properly cited.

\begin{abstract}
The construction of vertex-disjoint paths (disjoint paths) is an important research topic in various kinds of interconnection networks, which can improve the transmission rate and reliability. The $k$-ary $n$-cube is a family of popular networks. In this paper, we determine that there are $m(2 \leq m \leq n)$ disjoint paths in 3-ary $n$-cube covering $Q_{n}^{3}-F$ from $S$ to $T$ (many-to-many) with $|F| \leq 2 n-2 m$ and from $s$ to $T$ (one-to-many) with $|F| \leq 2 n-m-1$ where $s$ is in a fault-free cycle of length three.
\end{abstract}

\section{Introduction}

Vertex-disjoint paths (disjoint paths for short) are a set of paths in a graph that they do not share any vertices. A disjoint path cover of a graph is a disjoint path between two different vertices, and it covers all vertices of the graph. The disjoint path cover problem has been applied in many fields such as software testing, database design, and code optimization. Actually, vertex-disjoint paths can accelerate data transmission by providing parallel communication paths to avoid communication congestion. Besides, disjoint paths enhance the robustness of vertex failure and load balancing capability [1] and accelerate the transmission of large amounts of data by splitting data into disjoint communication paths of multiple vertices. The problem of many-tomany disjoint paths is an important problem in networks. It has attracted much attention because of its application in fault-tolerant routings for high-performance interconnection network.

Depending on the number of sources or sinks, there are one-to-one, one-to-many, and many-to-many disjoint paths problems. Among them, the one-to-one disjoint path is what we usually call the Hamilton path problem. A nonbipartite graph $G$ is one-to-one $m$-disjoint path coverable if there is an $m$-disjoint path cover between any two vertices of $G$. A bipartite graph is one-to-one $m$-disjoint path coverable if there is an $m$-disjoint path cover between any two vertices in different partite sets. Shih and Kao [1] constructed the oneto-one $m$-disjoint path cover of $k$-ary $n$-cube for $1 \leq m \leq 2 n$, $n \geq 2$ and $k \geq 3$. Li et al. [2] studied many-to-many $k$-disjoint paths in hypercubes with $f \leq 2 n-2 k-2,1 \leq k \leq n-1$ and each fault-free vertex has at least two fault-free neighbors. Chen in [3] considered many-to-many $k$ disjoint $(S, T)$-paths in the hypercube with $f_{v}+f_{e} \leq n-k-1$, $1 \leq k \leq n-1$ and any two sets $S$ and $T$ of $k$ fault-free vertices in different parts. Zhang and Wang [4] proved that many-tomany $m$ disjoint paths cover in $k$-ary $n$-cubes with even $k \geq 4$ and $1 \leq m \leq 2 n-1$. So, we consider some disjoint path problems about 3 -ary $n$-cubes. There is also research on other graphs. Such as one-to-many disjoint paths of $(n, k)$-star graph [5] and hyper-star networks [6] and oneto-one disjoint path covers on alternating group graphs [7].

For a set $S=\left\{s_{1}, s_{2}, \ldots, s_{k}\right\}$ of $k$ sources and a set $T=$ $\left\{t_{1}, t_{2}, \ldots, t_{k}\right\}$ of $k$ sinks in $V(G)$ with $S \cap T=\varnothing$, the manyto-many $k$-disjoint path problem is to determine whether there exist $k$ disjoint paths each joining a source and a sink. In other words, we need to find $k$ disjoint paths $P_{1}, P_{2}, \ldots, P_{k}$ in $G$ joining $S$ and $T$, which cover all the vertices in the graph; that is, $\cup_{1<i<k} V\left(P_{i}\right)=V(G)$ and $V\left(P_{i}\right) \cap V\left(P_{j}\right)=\varnothing$ for $i \neq j$ and $1 \leq i, j \leq k$. In addition, this problem is also subclassified into paired and unpaired types. In paired type, each source should be joined to a specific sink; that is, $s_{i}$ should be joined to $t_{i}$. Otherwise, each source can be joined to an arbitrary sink; that is, $s_{i}$ could be joined to 
$t_{j}$ for $i \neq j$. We study the unpaired type in this paper. Specially, if $S=\{s\}$, then it is called one-to-many disjoint paths; that is, $V\left(P_{i}\right) \cap V\left(P_{j}\right)=\{s\}$ for all $i \neq j$ and $1 \leq i, j \leq k$.

With the development of science and the expansion of the scale of computer system, people have more higher requirements for the reliability of network. However, computer failure is inevitable in a system. So, the disjoint path cover problem is also extended to a graph with some faulty elements $F$ (vertices and/or edges). A graph $G$ is called $f$-fault hamiltonian-connected if each pair of vertices are joined by a hamiltonian path in $G-F$ for any faulty elements $|F| \leq f$.

In this paper, we study $m$ disjoint paths of 3-ary $n$-cube covering $Q_{n}^{3}-F$ with faulty vertices $|F| \leq 2 n-2 m$ from $S$ to $T$ for $|S|=|T|=m$ in Theorem 1 . Besides, we also consider $m$ disjoint paths of 3 -ary $n$-cube covering $Q_{n}^{3}-F$ with faulty vertices $|F| \leq 2 n-m-1$ from $s$ to $T$ where $|T|=m$ and $s$ is in a fault-free cycle of length three in Theorem 2.

\section{Preliminaries}

2.1. Notations. An interconnection network can be represented by a graph $G=(V, E)$, where $V$ represents the vertex set and $E$ represents the edge set. Let $G$ be a simple undirected graph. For a vertex $v, N_{G}(v)$ is the set of vertices adjacent to $v$ in $G$. The path $P=\left\langle v_{1}, v_{2}, \ldots, v_{n-1}, v_{n}\right\rangle$ is a sequence of adjacent vertices where all the vertices are distinct, which is denoted by $P=\left(v_{1}, v_{n}\right)$. If the path $P$ satisfies $v_{1}=v_{n}$, it is called a cycle. In addition, $P^{-1}=$ $\left\langle v_{n}, v_{n-1}, \ldots, v_{2}, v_{1}\right\rangle$. For $1 \leq i<j \leq n, P\left[v_{i}, v_{j}\right]$ denotes the section from $v_{i}$ to $v_{j}$ in $P$. For $u v_{1}, v_{n} w \in E,\left\{u v_{1}\right\} \cup P \cup$ $\left\{v_{n} w\right\}=\left\langle u, v_{1}, \ldots, v_{n-1}, v_{n}, w\right\rangle$. A path or cycle which contains every vertex of a graph is called a Hamilton path (hamiltonian path) or Hamilton cycle (hamiltonian cycle) of the graph. A graph is hamiltonian if it contains a Hamilton cycle. A graph is hamiltonian-connected if there exists a Hamilton path between any two distinct vertices of the graph. For graph-theoretical terminology and notation not defined here, we follow [8].

\subsection{The k-Ary n-Cube $Q_{n}^{k}$}

Definition 1. The $k$-ary $n$-cube $Q_{n}^{k}(n \geq 1)$ is a graph with $k^{n}$ vertices, each of which has the form $u=u_{n-1} u_{n-2} \ldots u_{0}$, where $0 \leq u_{i} \leq k-1$ for every $0 \leq i \leq n-1$. Two vertices $u=$ $u_{n-1} u_{n-2} \ldots u_{0}$ and $v=v_{n-1} v_{n-2} \ldots v_{0}$ in $Q_{n}^{k}$ are adjacent if and only if there exists an integer $j, 0 \leq j \leq n-1$, such that $u_{j}=v_{j} \pm 1(\bmod k)$ and $u_{i}=v_{i}$ for every $i \in\{0,1, \ldots$, $n-1\} \backslash\{j\}$. For clarity of presentation, we omit writing " $(\bmod k)$ " in similar expressions for the remainder of the paper.

We discuss the 3 -ary $n$-cube $Q_{n}^{3}$ with $2 n$ regular. Obviously, $Q_{1}^{3}$ is a cycle of length 3 , and $Q_{2}^{3}$ is a $3 \times 3$ wraparound mesh. We can partition $Q_{n}^{3}$ along $j$-dimension, $0 \leq j \leq n-1$, by deleting all the $j$-dimension edges, into three disjoint subcubes, $Q_{n}^{3}[0], Q_{n}^{3}[1]$ and $Q_{n}^{3}[2]$ (abbreviated as $Q[0], Q[1]$ and $Q[2]$, if there are no ambiguities). $Q[j]$ is isomorphic to $Q_{n-1}^{3}$ for $0 \leq j \leq 2$. For $0 \leq i, j \leq 2$ and $i \neq j$, the edge set between $Q[i]$ and $Q[j]$ is a perfect matching.

Lemma 1 (see [9]). The $k$-ary $n$-cube is hamiltonian-connected when $k$ is odd.

Lemma 2 (see [10]). Assume $k$ is odd with $k \geq 3$. Let $F$ be a set of faulty vertices and/or edges in a k-ary n-cube $Q_{n}^{k}$.

(1) If $|F| \leq 2 n-2$, then the graph $Q_{n}^{k}-F$ is hamiltonian.

(2) If $|F| \leq 2 n-3$, then the graph $Q_{n}^{k}-F$ is hamiltonianconnected.

Lemma 3 (see [11]). Let $n \geq 2, F \subset V\left(Q_{n}^{3}\right),|F| \leq 2 n-4$. If $s_{1}, t_{1}, s_{2}, t_{2}$ are four vertices in $Q_{n}^{3}-F$, then there are two vertex-disjoint paths $P_{1}=\left(s_{1}, t_{1}\right)$ and $P_{2}=\left(s_{2}, t_{2}\right)$ such that $V\left(P_{1}\right) \cup V\left(P_{2}\right)=V\left(Q_{n}^{3}-F\right)$.

\section{Many-to-Many Disjoint Paths}

Theorem 1. Let $Q_{n}^{3}$ be a 3-ary $n$-cube for $n \geq 2$. Let $F$ be a vertex faulty set of $Q_{n}^{3}$. Let $S, T \subset V\left(Q_{n}^{3}\right)$ with $2 \leq|S|=|T|=m \leq n$. If $|F| \leq 2 n-2 m$, then there are $m$ disjoint paths from $S$ to $T$ covering $Q_{n}^{3}-F$.

Proof. We prove the theorem by induction on $m$. When $m=2,|S|=|T|=2$ and $|F| \leq 2 n-4$. By Lemma 3 , the theorem holds. Assume the theorem holds on $m-1$. We prove the theorem holds on $m$ for $n \geq m \geq 3$.

Suppose there is $s_{i} t_{j} \in E\left(Q_{n}^{3}\right)$ for $1 \leq i, j \leq m$. Note $\left|F \cup\left\{s_{i}, t_{j}\right\}\right| \leq 2 n-2 m+2=2 n-2(m-1)$. By the induction hypothesis, there are $(m-1)$ disjoint paths $P_{k}(1 \leq k \leq m-1) \quad$ from $S \backslash\left\{s_{i}\right\} \quad$ to $T \backslash\left\{t_{j}\right\} \quad$ covering $Q_{n}^{3}-F-\left\{s_{i}, t_{j}\right\}$. Let $P_{m}=\left\langle s_{i}, t_{j}\right\rangle$. Then, the theorem holds. Thus, we suppose $s_{i} t_{j} \notin E\left(Q_{n}^{3}\right)$ for all $1 \leq i, j \leq m$.

We decompose $Q_{n}^{3}$ into three isomorphic subgraphs $Q[0], Q[1], Q[2]$ along one dimension. Let $F_{i}=F \cap V$ (Q[i]), $S_{i}=S \cap V(Q[i])$ and $T_{i}=T \cap V(Q[i])$ for $0 \leq i \leq 2$. Let $S=\left\{s_{1}, \ldots, s_{m}\right\}$ and $T=\left\{t_{1}, \ldots, t_{m}\right\}$. If $s_{i}, t_{i} \in V(Q[j])$ for $1 \leq i \leq m$ and $0 \leq j \leq 2$, then let $s_{i}^{k}=N\left(s_{i}\right) \cap V(Q[k])$ and $t_{i}^{k}=N\left(t_{i}\right) \cap V(Q[k])$ for $k \in(\{0,1,2\} \backslash\{j\})$.

Case 1. $\left|S_{i}\right|=\left|T_{i}\right|$ for all $0 \leq i \leq 2$.

Case 1.1. $\left|S_{i}\right|=\left|T_{i}\right|=m$ for some $0 \leq i \leq 2$.

Without loss of generality, let $\left|S_{0}\right|=\left|T_{0}\right|=m$. Then, $\left|S_{1}\right|=\left|S_{2}\right|=\left|T_{1}\right|=\left|T_{2}\right|=0$. Let $S_{0}=\left\{s_{1}, \ldots, s_{m}\right\}$ and $T_{0}=\left\{t_{1}, \ldots, t_{m}\right\}$.

Case 1.1.1. $\left|F_{0}\right|=2 n-2 m$.

Then, $\left|F_{1}\right|=\left|F_{2}\right|=0$. Let $S_{0}^{\prime}=S_{0} \backslash\left\{s_{m}\right\}$ and $T_{0}^{\prime}=$ $T_{0} \backslash\left\{t_{m}\right\}$. Then, $\left|S_{0}^{\prime}\right|=\left|T_{0}^{\prime}\right|=m-1$. By the induction hypothesis, there are $(m-1)$ disjoint paths $R_{i}=\left(s_{i}, t_{i}\right)(1 \leq i \leq m-1)$ from $S_{0}^{\prime}$ to $T_{0}^{\prime}$ covering $Q[0]-F_{0}-s_{m}-t_{m}$. Then, $s_{m}, t_{m} \in \cup_{i=1}^{m-1} V\left(R_{i}\right)$.

Case 1.1.1.1. $s_{m}, t_{m} \in V\left(R_{i}\right)$ for some $1 \leq i \leq m-1$.

Without loss of generality, let $s_{m}, t_{m} \in V\left(R_{1}\right)$. Suppose $s_{m}$ is before $t_{m}$ in $R_{1}$. Then, $R_{1}=R_{1}\left[s_{1}, s_{m}\right] \cup$ $R_{1}\left[s_{m}, t_{m}\right] \cup R_{1}\left[t_{m}, t_{1}\right]$. Let $u$ (resp. $v$ ) be the before 
vertex (resp. later vertex) of $s_{m}$ (resp. $\left.t_{m}\right)$ in $R_{1}$. Let $u_{1}=$ $N(u) \cap V(Q[1])$ and $v_{1}=N(v) \cap V(Q[1])$. By Lemma 1 , there is a Hamilton path $L=\left(u_{1}, v_{1}\right)$ in $Q[1]$. Let $x y \in E(L), \quad x_{2}=N(x) \cap V(Q[2]) \quad$ and $y_{2}=N(y) \cap V(Q[2])$. By Lemma 1, there is a Hamilton path $M=\left(x_{2}, y_{2}\right)$ in $Q[2]$. Let $P_{1}=R_{1}\left[s_{1}, u\right] \cup$ $\left\{u u_{1}, v v_{1}\right\} \cup(L-x y) \cup\left\{x x_{2}, y y_{2}\right\} \cup M \cup R_{1}\left[v, t_{1}\right], P_{i}=$ $R_{i}(2 \leq i \leq m-1)$ and $P_{m}=R_{1}\left[s_{m}, t_{m}\right]$. Similarly, $t_{m}$ is before $s_{m}$ in $R_{1}$.

Case 1.1.1.2. $s_{m} \in V\left(R_{i}\right), t_{m} \in V\left(R_{j}\right)$ for $1 \leq i, j \leq m-1$ and $i \neq j$.

Without loss of generality, let $s_{m} \in V\left(R_{1}\right)$ and $t_{m} \in V\left(R_{2}\right)$. Let $u$ and $v$ be the before and later vertex of $s_{m}$ in $R_{1}$, respectively. Let $u_{1}=N(u) \cap V(Q[1])$ and $v_{1}=N(v) \cap V(Q[1])$. Let $x$ and $y$ be the before and later vertex of $t_{m}$ in $R_{2}$, respectively. Let $x_{1}=$ $N(x) \cap V(Q[1])$ and $y_{1}=N(y) \cap V(Q[1])$. Note $\left|F_{1}\right|=0 \leq 2 n-6$ for $n \geq 3$. By Lemma 3 , there are two disjoint paths $L_{1}=\left(u_{1}, v_{1}\right)$ and $L_{2}=\left(x_{1}, y_{1}\right)$ covering $Q[1]-F_{1}$. By Lemma 1 , there is a Hamilton path $M=$ $\left(s_{m}^{2}, t_{m}^{2}\right)$ in $Q$ [2]. Let $P_{1}=\left(R_{1}-s_{m}\right) \cup\left\{u u_{1}, v v_{1}\right\} \cup L_{1}$, $P_{2}=\left(R_{2}-t_{m}\right) \cup\left\{x x_{1}, y y_{1}\right\} \cup L_{2}, P_{i}=R_{i}(3 \leq i \leq m-1)$ and $P_{m}=\left\{s_{m} s_{m}^{2}, t_{m} t_{m}^{2}\right\} \cup M$.

Case 1.1.2. $\left|F_{0}\right|=2 n-2 m-1$.

Then, $\left|F_{1} \cup F_{2}\right| \leq 1$. Without loss of generality, let $\left|F_{1}\right|=$ 0 and $\left|F_{2}\right| \leq 1$. Let $S_{0}^{\prime}=S_{0} \backslash\left\{s_{m}\right\}$ and $T_{0}^{\prime}=T_{0} \backslash\left\{t_{m}\right\}$. Then, $\left|S_{0}^{\prime}\right|=\left|T_{0}^{\prime}\right|=m-1$. Note $\left|F_{0} \cup\left\{t_{m}\right\}\right|=2 n-2 m$. By the induction hypothesis, there are $(m-1)$ disjoint paths $R_{i}=\left(s_{i}, t_{i}\right)(1 \leq i \leq m-1)$ from $S_{0}^{\prime}$ to $T_{0}^{\prime}$ covering $\mathrm{Q}[0]-F_{0}-t_{m}$. Then, $s_{m} \in V\left(R_{i}\right)$ for some $1 \leq i \leq$ $m-1$. Without loss of generality, let $s_{m} \in V\left(R_{1}\right)$. Then, $R_{1}=R_{1}\left[s_{1}, s_{m}\right] \cup R_{1}\left[s_{m}, t_{1}\right]$. Let $u$ and $v$ be the before and later vertex of $s_{m}$ in $R_{1}$, respectively. Let $u_{i}=N(u) \cap V(Q[i])$ and $v_{i}=N(v) \cap V(Q[i])$ for $1 \leq i \leq 2$.

If $u_{2} \in F_{2}$ or $v_{2} \in F_{2}$, then $s_{m}^{2} \notin F_{2}$ and $t_{m}^{2} \notin F_{2}$. By Lemma 2 , there is a path $L_{1}=\left(u_{1}, v_{1}\right)$ and $M_{1}=$ $\left(s_{m}^{2}, t_{m}^{2}\right)$ covering $Q[1]-F_{1}$ and $Q[2]-F_{2}$, respectively. Let $P_{1}=\left(R_{1}-s_{m}\right) \cup\left\{u u_{1}, v v_{1}\right\} \cup L_{1}, P_{i}=R_{i}(2 \leq$ $i \leq m-1)$ and $P_{m}=\left\{s_{m} s_{m}^{2}, t_{m} t_{m}^{2}\right\} \cup M_{1}$.

Suppose $u_{2} \notin F_{2}$ and $v_{2} \notin F_{2}$. By Lemma 2, there is a path $L_{2}=\left(s_{m}^{1}, t_{m}^{1}\right)$ and $M_{2}=\left(u_{2}, v_{2}\right)$ covering $Q[1]-F_{1}$ and $Q[2]-F_{2}$, respectively. Let $P_{1}=\left(R_{1}-s_{m}\right) \cup$ $\left\{u u_{2}, v v_{2}\right\} \cup M_{2}, \quad P_{i}=R_{i}(2 \leq i \leq m-1)$ and $P_{m}=\left\{s_{m} s_{m}^{1}\right.$, $\left.t_{m} t_{m}^{1}\right\} \cup L_{2}$.

Case 1.1.3. $2 n-2 m-3 \leq\left|F_{0}\right| \leq 2 n-2 m-2$.

In this case, $2 n-2 m-2 \geq 0$. We get $n \geq m+1 \geq 4$ for $m \geq 3$. We have $\left|F_{1} \cup F_{2}\right| \leq 3$. Without loss of generality, let $\left|F_{1}\right| \leq\lfloor 3 / 2\rfloor=1$. Since $|S|=|T|=m \geq 3>1 \geq\left|F_{1}\right|$, there are two vertices $s_{i}^{1}, t_{i}^{1} \notin F_{1}$ for some $1 \leq i \leq m$. Without loss of generality, let $s_{m}^{1}, t_{m}^{1} \notin F_{1}$. Let $S_{0}^{\prime}=S_{0} \backslash\left\{s_{m}\right\}$ and $T_{0}^{\prime}=T_{0} \backslash\left\{t_{m}\right\}$. Then, $\left|S_{0}^{\prime}\right|=\left|T_{0}^{\prime}\right|=$ $m-1$. Note $\left|F_{0} \cup\left\{s_{m}, t_{m}\right\}\right| \leq 2 n-2 m$. By the induction hypothesis, there are $(m-1)$ disjoint paths $P_{i}=\left(s_{i}, t_{i}\right)(1 \leq i \leq m-1)$ from $S_{0}^{\prime}$ to $T_{0}^{\prime}$ covering $Q[0]-F_{0}-s_{m}-t_{m}$. Since $\quad|V(Q[1])|-\left|F_{1} \cup F_{2}\right|-$ $\left|\left\{s_{m}^{1}, t_{m}^{1}\right\}\right| \geq 3^{n-1}-3-2 \geq 4$ for $n \geq 3$, there are two edges $x_{1} x_{2}$ and $y_{1} y_{2}$ such that $\left\{x_{i}, y_{i}\right\} \subset V\left(Q[i]-F_{i}-\right.$ $\left.\left\{s_{m}^{1}, t_{m}^{1}\right\}\right)$ for $1 \leq i \leq 2$. Note $\left|F_{1}\right| \leq 1 \leq 2 n-6$ for $n \geq 4$. By Lemma 3, there are two disjoint paths $L_{1}=\left(s_{m}^{1}, x_{1}\right)$ and $L_{2}=\left(y_{1}, t_{m}^{1}\right)$ covering $Q[1]-F_{1}$. Note $\left|F_{2}\right| \leq 3 \leq 2 n-5$ for $n \geq 4$. By Lemma 2, there is a path $M=\left(x_{2}, y_{2}\right)$ covering $Q[2]-F_{2}$. Let $P_{m}=\left\{s_{m} s_{m}^{1}\right\} \cup L_{1} \cup\left\{x_{1} x_{2}\right\} \cup$ $M \cup\left\{y_{2} y_{1}\right\} \cup L_{2} \cup\left\{t_{m}^{1} t_{m}\right\}$.

Case 1.1.4. $\left|F_{0}\right| \leq 2 n-2 m-4$.

Let $N_{Q[0]}\left(s_{m}\right)=\left\{u_{1}, \ldots, u_{2 n-2}\right\}$ and $u_{i}^{j}=N\left(u_{i}\right) \cap$ $V(Q[j])$ for $1 \leq i \leq 2 n-2$ and $j=1,2$. Since $\left|N_{Q[0]}\left(s_{m}\right)\right|-\left|S \backslash\left\{s_{m}\right\}\right|=2 n-2-(m-1)>2 n-2 m \geq$ $|F|$ for $m \geq 3$, there is an edge $u_{i} u_{i}^{1}$ such that $u_{i} \in V\left(Q[0]-F_{0}-S\right)$ and $u_{i}^{1} \in V\left(Q[1]-F_{1}\right)$ for $1 \leq i \leq 2 n-2$. Without loss of generality, let $u_{1} u_{1}^{1}$ be satisfied. Let $N_{\mathrm{Q}[0]}\left(t_{i}\right)=\left\{v_{1}, \ldots, v_{2 n-2}\right\} \quad$ and $v_{i}^{j}=N\left(v_{i}\right) \cap V(Q[j])$ for $1 \leq i \leq 2 n-2$ and $j=1,2$. Since $\left|N_{Q[0]}\left(t_{m}\right)\right|-\left|T \backslash\left\{t_{m}\right\}\right|-\left|\left\{u_{1}\right\}\right| \geq 2 n-2-(m-1)-$ $1>2 n-2 m \geq|F|$ for $m \geq 3$, there is an edge $v_{i} v_{i}^{1}$ such that $v_{i} \in V\left(Q[0]-F_{0}-u_{1}\right)$ and $v_{i}^{1} \in V\left(Q[1]-F_{1}-u_{1}^{1}\right)$ for $1 \leq i \leq 2 n-2$. Without loss of generality, let $v_{1} v_{1}^{1}$ be satisfied.

Note $\left|F_{0} \cup\left\{s_{m}, t_{m}, u_{1}, v_{1}\right\}\right| \leq 2 n-2 m$. By the induction hypothesis, there are $(m-1)$ disjoint paths $P_{i}=\left(s_{i}, t_{i}\right)(1 \leq i \leq m-1)$ covering. Since $|V(Q[1])|-$ $|F|-\left|\left\{u_{1}^{1}, v_{1}^{1}\right\}\right| \geq 3^{n-1}-(2 n-2 m)-2 \geq 7$ for $3 \leq m \leq n$, there are two edges $x_{1} x_{2}, y_{1} y_{2}$ such that $\left\{x_{i}, y_{i}\right\} \subset V\left(Q[i]-F_{i}-\left\{u_{1}^{1}, v_{1}^{1}\right\}\right)$ for $i=1,2$. Note $\left|F_{1}\right| \leq 2 n-2 m \leq 2 n-6$ for $m \geq 3$. By Lemma 3 , there are two disjoint paths $L_{1}=\left(u_{1}^{1}, x_{1}\right)$ and $L_{2}=\left(y_{1}, v_{1}^{1}\right)$ covering $Q[1]-F_{1}$. Note $\left|F_{2}\right| \leq 2 n-2 m<2 n-5$ for $m \geq 3$. By Lemma 2 , there is a path $M=\left(x_{2}, y_{2}\right)$ covering $Q[2]-F_{2}$. Let $P_{m}=\left\{s_{m} u_{1}, u_{1} u_{1}^{1}\right.$, $\left.t_{m} v_{1}, v_{1} v_{1}^{1}\right\} \cup L_{1} \cup L_{2} \cup\left\{x_{1} x_{2}, y_{1} y_{2}\right\} \cup M$.

Case 1.2. $\left|S_{i}\right|=\left|T_{i}\right|=m-1$ for some $0 \leq i \leq 2$.

Without loss of generality, let $\left|S_{0}\right|=\left|T_{0}\right|=1$, $\left|S_{1}\right|=\left|T_{1}\right|=m-1$ and $\left|S_{2}\right|=\left|T_{2}\right|=0$. Let $S_{0}=\left\{s_{1}\right\}$, $T_{0}=\left\{t_{1}\right\}, S_{1}=\left\{s_{2}, \ldots, s_{m}\right\}$ and $T_{1}=\left\{t_{2}, \ldots, t_{m}\right\}$. By the induction hypothesis, there are $(m-1)$ disjoint paths $P_{i}=\left(s_{i}, t_{i}\right)(2 \leq i \leq m)$ covering $Q[1]-F_{1}$. Since $|V(Q[i])|-|F|-\left|S_{0}\right|-\left|T_{0}\right| \geq 3^{n-1}-(2 n-2 m)-2 \geq 7$ for $3 \leq m \leq n$, there are two edges $x_{1} x_{2}, y_{1} y_{2}$ such that $\left\{x_{i}, y_{i}\right\} \subset V\left(Q[i]-F_{i}-S_{0}-T_{0}\right)$ for $i=0,2$. Note $\left|F_{0}\right| \leq 2 n-2 m \leq 2 n-6$ for $m \geq 3$. By Lemma 3 , there are two disjoint paths $R_{1}=\left(s_{1}, x_{0}\right)$ and $R_{2}=\left(y_{0}, t_{1}\right)$ covering $Q[0]-F_{0}$. By Lemma 2 , there is a path $M=$ $\left(x_{2}, y_{2}\right)$ covering $Q[2]-F_{2}$. Let $P_{1}=R_{1} \cup\left\{x_{0} x_{2}\right\} \cup$ $M \cup\left\{y_{0} y_{2}\right\} \cup R_{2}$.

Case 1.3. $1 \leq\left|S_{i}\right|=\left|T_{i}\right| \leq m-2$ for all $0 \leq i \leq 2$.

Let $\left|S_{i}\right|=\left|T_{i}\right|=p_{i} \leq m-2$ for $0 \leq i \leq 2$. Then, $p_{0}+p_{1}+p_{2}=m$. Note $\left|F_{i}\right| \leq 2 n-2 m \leq 2(n-1)-2 p_{i}$. By the induction hypothesis, there are $p_{i}$ disjoint paths covering $Q[i]-F_{i}$ for $0 \leq i \leq 2$.

Case 1.4. $\left|S_{i}\right|=\left|T_{i}\right|=0$ for only one $0 \leq i \leq 2$.

Let $\left|S_{0}\right|=\left|T_{0}\right|=0$. If there is $\left|S_{i}\right|=\left|T_{i}\right|=m-1$ or $\left|S_{i}\right|=$ $\left|T_{i}\right|=1$ for some $1 \leq i \leq 2$, then it is similar to Case 1.2. 
Thus, suppose $2 \leq\left|S_{i}\right|=\left|T_{i}\right| \leq m-2$ for $1 \leq i \leq 2$. Let $\left|S_{1}\right|=\left|T_{1}\right|=p$. Then, $\left|S_{2}\right|=\left|T_{2}\right|=m-p$. Let $S_{1}=\left\{s_{1}, \ldots, s_{p}\right\}, T_{1}=\left\{t_{1}, \ldots, t_{p}\right\}, S_{2}=\left\{s_{p+1}, \ldots, s_{m}\right\}$ and $T_{2}=\left\{t_{p+1}, \ldots, t_{m}\right\}$. Note $\left|S_{1}\right|=\left|T_{1}\right|=p \leq m-2$. By the induction hypothesis, there are $p$ disjoint paths $P_{i}=\left(s_{i}, t_{i}\right)(1 \leq i \leq p)$ covering $Q[1]-F_{1}$.

Since $|V(Q[i])|-|F|-|S|-|T| \geq 3^{n-1}-(2 n-2 m)-$ $2 m \geq 3$ for $n \geq 3$, there are two edges $u_{0} u_{2}, v_{0} v_{2}$ such that $u_{i}, v_{i} \in V(Q[i]-F-S-T)$ for $i=0,2$. Note $\left|S_{2} \cup\left\{v_{2}\right\}\right|=\left|T_{2} \cup\left\{u_{2}\right\}\right|=m-p+1 \leq m-1$. By the induction hypothesis, there are $(m-p+1)$ disjoint paths $P_{i}=\left(s_{i}, t_{i}\right)(p+1 \leq i \leq m-1), M_{1}=\left(s_{m}, u_{2}\right)$ and $M_{2}=\left(v_{2}, t_{m}\right) \quad$ covering $\quad Q[2]-F_{2}$. Note $\left|F_{0}\right| \leq 2 n-2 m<2 n-5$ for $m \geq 3$. By Lemma 2 , there is a path $R=\left(u_{0}, v_{0}\right)$ covering $Q[0]-F_{0}$. Let $P_{m}=M_{1} \cup\left\{u_{2} u_{0}\right\} \cup R \cup\left\{v_{0} v_{2}\right\} \cup M_{2}$.

Case 2. $\left|S_{i}\right|=\left|T_{i}\right|$ for only one $0 \leq i \leq 2$.

Without loss of generality, let $\left|S_{0}\right|=\left|T_{0}\right|$. Then, $\left|S_{1}\right| \neq\left|T_{1}\right|, \quad\left|S_{2}\right| \neq\left|T_{2}\right|$ and $\left|S_{1}\right|+\left|S_{2}\right|=\left|T_{1}\right|+\left|T_{2}\right|$. Without loss of generality, let $\left|S_{1}\right|>\left|T_{1}\right|$. Then, $\left|S_{2}\right|<\left|T_{2}\right|$ and $\left|S_{1}\right|-\left|T_{1}\right|=\left|T_{2}\right|-\left|S_{2}\right|$. Since $|V(Q[i])|-$ $|F|-|S|-|T| \geq 3^{n-1}-(2 n-2 m)-2 m \geq m$ for $3 \leq$ $m \leq n$, there are $m$ cycles of length three $C_{i}=\left\langle w_{i}^{0}, w_{i}^{1}\right.$, $\left.w_{i}^{2}, w_{i}^{0}\right\rangle$ for $1 \leq i \leq m$ such that $w_{i}^{j} \in V\left(Q[j]-F_{j}-S-\right.$ T) for $0 \leq j \leq 2$.

Case 2.1. $\left|S_{0}\right|=\left|T_{0}\right|=0$.

Then, $\left|S_{1}\right|+\left|S_{2}\right|=\left|T_{1}\right|+\left|T_{2}\right|=m$.

Case 2.1.1. $\left|S_{1}\right|=m$ and $\left|T_{1}\right|=0$.

Then, $\left|S_{2}\right|=0$ and $\left|T_{2}\right|=m$. Let $S_{1}=\left\{s_{1}, \ldots, s_{m}\right\}$, $T_{2}=\left\{t_{1}, \ldots, t_{m}\right\}$.

Case 2.1.1.1. $\left|F_{0}\right|=0$.

Let $S_{0}^{\prime}=\left\{s_{i}^{0}: 1 \leq i \leq m-1\right\}, \quad T_{0}^{\prime}=\left\{t_{i}^{0}: 1 \leq i \leq m-1\right\}$. Then, $\left|S_{0}^{\prime}\right|=\left|T_{0}^{\prime}\right|=m-1$. By the induction hypothesis, there are $(m-1)$ disjoint paths $R_{i}=\left(s_{i}^{0}, t_{i}^{0}\right)(1 \leq i \leq m-$ 1) from $S_{0}^{\prime}$ to $T_{0}^{\prime}$ covering $Q[0]-F_{0}$. Let $P_{i}=\left\{s_{i} s_{i}^{0}\right\} \cup$ $R_{i} \cup\left\{t_{i}^{0} t_{i}\right\}(1 \leq i \leq m-1)$.

Let $W_{1}=\left\{w_{1}^{1}\right\}$ and $W_{2}=\left\{w_{1}^{2}\right\}$. Note $\left|S_{1}^{\prime}\right|=\left|S_{1} \backslash\left\{s_{m}\right\}\right|=$ $m-1=\left|T_{2} \backslash\left\{t_{m}\right\}\right|=\left|T_{2}^{\prime}\right|$ and $\left|F_{1}\right|+\left|S_{1}^{\prime}\right| \leq 2 n-2 m+$ $(m-1)=2 n-m-1 \leq 2 n-5$ for $m \geq 4$. By Lemma 2 , there is a path $L=\left(s_{m}, w_{1}^{1}\right)$ covering $Q[1]-F_{1}$. Similarly, there is a path $M=\left(w_{1}^{2}, t_{m}\right)$ covering $Q[2]-F_{2}$. Let $P_{m}=L \cup\left\{w_{1}^{1} w_{1}^{2}\right\} \cup M$.

Suppose $m=3$. Then, $|F| \leq 2 n-2 m=2 n-6$. Let $W_{1}=$ $\left\{w_{1}^{1}, w_{2}^{1}\right\}$ and $W_{2}=\left\{w_{1}^{2}, w_{2}^{2}\right\}$. By Lemma 2, there are two paths $L_{1}=\left(s_{1}, w_{1}^{1}\right)$ and $L_{2}=\left(s_{2}, w_{2}^{1}\right)$ (resp. $M_{1}=$ $\left(w_{2}^{1}, t_{1}\right)$ and $\left.M_{2}=\left(w_{2}^{2}, t_{2}\right)\right)$ covering $Q[1]-F_{1}$ (resp. $\left.Q[2]-F_{2}\right)$. Let $P_{i}^{\prime}=L_{i} \cup\left\{w_{i}^{1} w_{i}^{2}\right\} \cup M_{i}$ for $i=1,2$. Then, $s_{3}, t_{3} \in P_{i}^{\prime}$ for $1 \leq i \leq 2$.

Suppose $s_{3}, t_{3} \in P_{1}^{\prime}$. Let $P_{3}=P_{1}^{\prime}\left[s_{3}, t_{3}\right]$. Let $u$ and $v$ be the before and later vertex of $s_{3}$ and $t_{3}$, respectively. Let $u_{0}=N(u) \cap V(Q[0])$ and $v_{0}=N(v) \cap V(Q[0])$.

If $u_{0}=v_{0}$, then let $P_{1}=P_{1}^{\prime}\left[s_{1}, u\right] \cup\left\{u u_{0}, v_{0} v\right\} \cup$ $P_{1}^{\prime}\left[v, t_{1}\right]$. Let $w z \in P_{2}^{\prime}, w_{0}=N(w) \cap V(Q[0])$ and $z_{0}=$ $N(z) \cap V(Q[0])$. Note $\left|F_{0} \cup\left\{u_{0}\right\}\right|=1 \leq 2 n-5$ for $n \geq 3$. By Lemma 2 , there is a path $R=\left(w_{0}, z_{0}\right)$ covering $Q[0]-F_{0}-u_{0}$. Let $P_{2}=\left(P_{2}^{\prime}-w z\right) \cup\left\{w w_{0}, z z_{0}\right\} \cup R$.

Suppose $u_{0} \neq v_{0}$. Note $\left|F_{0}\right|=0$. By Lemma 1 , there is a Hamilton path $R=\left(u_{0}, v_{0}\right)$ in $Q[0]$. Let $P_{1}=P_{1}^{\prime}\left[s_{1}, u\right] \cup\left\{u u_{0}, v v_{0}\right\} \cup R \cup P_{1}^{\prime}\left[v, t_{1}\right]$ and $P_{2}=P_{2}^{\prime}$.

Suppose $s_{3} \in P_{1}^{\prime}$ and $t_{3} \in P_{2}^{\prime}$. Let $u$ and $v$ be the before and later vertex of $s_{3}$ and $t_{3}$ in $P_{1}^{\prime}$ and $P_{2}^{\prime}$, respectively. Let $u_{0}=N(u) \cap V(Q[0])$ and $v_{0}=N(v) \cap V(Q[0])$.

If $u_{0}=v_{0}$, then let $P_{1}=P_{1}^{\prime}\left[s_{1}, u\right] \cup\left\{u u_{0}, v_{0} v\right\} \cup$ $P_{2}^{\prime}\left[v, t_{2}\right]$. Let $w z \in L_{2}, w_{0}=N(w) \cap V(Q[0])$ and $z_{0}=$ $N(z) \cap V(Q[0])$. Note $\left|F_{0} \cup\left\{u_{0}\right\}\right|=1 \leq 2 n-5$ for $n \geq 3$. By Lemma 2 , there is a path $R=\left(w_{0}, z_{0}\right)$ covering $Q[0]-F_{0}-u_{0}$. Let $\quad P_{2}=\left(P_{2}^{\prime}\left[s_{2}, t_{3}\right]-w z\right) \cup$ $\left\{w w_{0}, z z_{0}\right\} \cup R$ and $P_{3}=P_{1}^{\prime}\left[s_{3}, t_{1}\right]$.

Suppose $u_{0} \neq v_{0}$. Note $\left|F_{0}\right|=0$. By Lemma 1 , there is a Hamilton path $R=\left(u_{0}, v_{0}\right)$ in $Q[0]$. Let $P_{1}=P_{1}^{\prime}\left[s_{1}, u\right] \cup\left\{u u_{0}, v v_{0}\right\} \cup R \cup P_{2}^{\prime}\left[v, t_{2}\right], P_{2}=P_{2}^{\prime}\left[s_{2}\right.$, $\left.t_{3}\right]$ and $P_{3}=P_{1}^{\prime}\left[s_{3}, t_{1}\right]$.

Case 2.1.1.2. $\left|F_{0}\right|=1$.

There is at most one vertex $s_{i}^{0} \in F_{0}$ or $t_{i}^{0} \in F_{0}$ for $1 \leq i \leq m$. Without loss of generality, let $s_{i}^{0}, t_{i}^{0} \notin F_{0}$ for all $1 \leq i \leq m-1$. Let $S_{0}^{\prime}=\left\{s_{i}^{0}: 1 \leq i \leq m-2\right\}, T_{0}^{\prime}=\left\{t_{i}^{0}: 1 \leq\right.$ $i \leq m-2\}$. Then, $\left|S_{0}^{\prime}\right|=\left|T_{0}^{\prime}\right|=m-2$. Note $\left|F_{0}\right|=$ $1 \leq 2(n-1)-2(m-2)$. By the induction hypothesis, there are $(m-2)$ disjoint paths $R_{i}=\left(s_{i}^{0}, t_{i}^{0}\right)(1 \leq i \leq m-$ 2) from $S_{0}^{\prime}$ to $T_{0}^{\prime}$ covering $Q[0]-F_{0}$. Let $P_{i}=\left\{s_{i} s_{i}^{0}\right\} \cup$ $R_{i} \cup\left\{t_{i}^{0} t_{i}\right\}(1 \leq i \leq m-2)$.

Let $W_{1}=\left\{w_{1}^{1}, w_{2}^{1}\right\}$ and $W_{2}=\left\{w_{1}^{2}, w_{2}^{2}\right\}$. Note $\left|S_{1}^{\prime}\right|=\left|S_{1}\right|$ $\left\{s_{m-1}, s_{m}\right\}|=m-2=| T_{2} \backslash\left\{t_{m-1}, t_{m}\right\}|=| T_{2}^{\prime} \mid$ and $\left|F_{1}\right|+$ $\left|S_{1}^{\prime}\right| \leq 2 n-2 m-1+(m-2)=2 n-m-3 \leq 2 n-6$ for $m \geq 3$. By Lemma 3 , there are two disjoint paths $L_{1}=$ $\left(s_{m-1}, w_{1}^{1}\right)$ and $L_{2}=\left(s_{m}, w_{2}^{1}\right)$ covering $Q[1]-F_{1}$. Similarly, there are two disjoint paths $M_{1}=\left(w_{1}^{2}, t_{m-1}\right)$ and $M_{2}=\left(w_{2}^{2}, t_{m}\right)$ covering $Q[2]-F_{2}$. Let $P_{m-1}=L_{1} \cup\left\{w_{1}^{1} w_{1}^{2}\right\} \cup M_{1}$ and $P_{m}=L_{2} \cup\left\{w_{2}^{1} w_{2}^{2}\right\} \cup M_{2}$.

Case 2.1.1.3. $\left|F_{0}\right| \geq 2$.

Then, $\left|F_{i}\right| \leq 2 n-2 m-2$ for $i=1,2$. Let $N_{Q[1]}\left(s_{m}\right)=$ $\left\{u_{1}, \ldots, u_{2 n-2}\right\}$ and $u_{i}^{0}=N\left(u_{i}\right) \cap V(Q[0])$ for $1 \leq i \leq$ $2 n-2$. Since $\left|N_{Q[1]}\left(s_{m}\right)\right|-\left|S \backslash\left\{s_{m}\right\}\right|=2 n-2-(m-$ 1) $>2 n-2 m \geq|F|$ for $m \geq 3$, there is an edge $u_{i} u_{i}^{0}(1 \leq i \leq 2 n-2)$ such that $u_{i} \in V\left(Q[1]-F_{1}-S_{1}\right)$ and $u_{i}^{0} \in V\left(Q[0]-F_{0}\right)$. Without loss of generality, let $u_{1} u_{1}^{0}$ be satisfied. Let $N_{Q[2]}\left(t_{m}\right)=\left\{v_{1}, \ldots, v_{2 n-2}\right\}$ and $v_{i}^{0}=N\left(v_{i}\right) \cap V(\mathrm{Q}[0])$ for $1 \leq i \leq 2 n-2$. Since $\left|N_{Q[2]}\left(t_{m}\right)\right|-\left|T \backslash\left\{t_{m}\right\}\right|=2 n-2-(m-1)>2 n-2 m+$ $1 \geq|F|+\left|\left\{u_{1}^{0}\right\}\right|$ for $m \geq 3$, there is an edge $v_{i} v_{i}^{0}$ such that $v_{i} \in V\left(Q[2]-F_{2}-T_{2}\right)$ and $v_{i}^{0} \in V\left(Q[0]-F_{0}-\left\{u_{1}^{0}\right\}\right)$. Without loss of generality, let $v_{1} v_{1}^{0}$ be satisfied. Let $S_{1}^{\prime}=$ $S_{1} \backslash\left\{s_{m}\right\}$ and $T_{2}^{\prime}=T_{2} \backslash\left\{t_{m}\right\}$. Then, $\left|S_{1}^{\prime}\right|=\left|T_{2}^{\prime}\right|=m-1$. Let $W_{1}=\left\{w_{i}^{1}: 1 \leq i \leq m-1\right\}$ and $W_{2}=\left\{w_{i}^{2}: 1 \leq i \leq\right.$ $m-1\}$. Note $\left|F_{1} \cup\left\{s_{m}, u_{1}\right\}\right| \leq 2 n-2 m$ and $\mid F_{2} \cup\left\{t_{m}\right.$, $\left.v_{1}\right\} \mid \leq 2 n-2 m$. By the induction hypothesis, there are $(m-1)$ disjoint paths $L_{i}=\left(s_{i}, w_{i}^{1}\right)\left(\operatorname{resp} . M_{i}=\left(w_{i}^{2}, t_{i}\right)\right)$ $(1 \leq i \leq m-1)$ from $S_{1}^{\prime}$ to $W_{1}$ (resp. from $W_{2}$ to $T_{2}^{\prime}$ ) covering $Q[1]-F_{1}-s_{m}-u_{1}$ (resp. $Q[2]-F_{2}-t_{m}-$ 
$\left.v_{1}\right)$. Note $\left|F_{0}\right| \leq 2 n-2 m<2 n-5$ for $m \geq 3$. By Lemma 2 , there is a path $R=\left(u_{1}^{0}, v_{1}^{0}\right)$ covering $Q[0]-F_{0}$. Let $P_{i}=L_{i} \cup\left\{w_{i}^{1} w_{i}^{2}\right\} \cup M_{i}(1 \leq i \leq m-1)$ and $P_{m}=\left\{s_{m} u_{1}\right.$, $\left.u_{1} u_{1}^{0}, t_{m} v_{1}, v_{1} v_{1}^{0}\right\} \cup R$.

Case 2.1.2. $\left|S_{1}\right|=m$ and $1 \leq\left|T_{1}\right| \leq m-1$.

Let $S_{1}=\left\{s_{1}, \ldots, s_{m}\right\}$. Let $\left|T_{1}\right|=p \leq m-1$. Then, $\left|T_{2}\right|=m-p \leq m-1$. Let $T_{1}=\left\{t_{1}, \ldots, t_{p}\right\} \quad$ and $T_{2}=\left\{t_{p+1}, \ldots, t_{m}\right\}$. Let $W_{2}=\left\{w_{i}^{2}: p+1 \leq i \leq m\right\}$. Note $\left|W_{2}\right|=\left|T_{2}\right|=m-p \leq m-1$. By the induction hypothesis, there are $(m-p)$ disjoint paths $M_{i}=\left(w_{i}^{2}, t_{i}\right)(p+1 \leq i \leq m)$ covering $Q[2]-F_{2}$.

Case 2.1.2.1. $\left|F_{0}\right|=0$.

Let $W_{1}=\left\{w_{i}^{1}: p+1 \leq i \leq m-1\right\}$ for $p \leq m-2, W_{1}=\varnothing$ for $p=m-1$. Then, $\left|W_{1}\right|=m-p-1$. Note $\left|T_{1}\right|+\left|W_{1}\right|=p+(m-p-1)=m-1=\left|S_{1} \backslash\left\{s_{m}\right\}\right|$. By the induction hypothesis, there are $(m-1)$ disjoint paths $L_{i}=\left(s_{i}, t_{i}\right)(1 \leq i \leq p)$ and $L_{i}=\left(s_{i}, w_{i}^{1}\right)(p+$ $1 \leq i \leq m-1)$ covering $Q[1]-F_{1}$. Let $P_{i}^{\prime}=L_{i}(1 \leq i \leq p)$, $P_{i}^{\prime}=L_{i} \cup\left\{w_{i}^{1} w_{i}^{2}\right\} \cup M_{i}(p+1 \leq i \leq m-1)$. Then, $s_{m} \in$ $V\left(P_{j}^{\prime}\right)$ for some $1 \leq j \leq m-1$. We get $P_{j}^{\prime}=$ $P_{j}^{\prime}\left[s_{j}, s_{m}\right] \cup P_{j}^{\prime}\left[s_{m}, t_{j}\right]$. Let $u$ and $v$ be the before and later vertex of $s_{m}$ in $P_{j}^{\prime}$, respectively. Let $u_{0}=N(u) \cap$ $V(Q[0]), v_{0}=N(v) \cap V(Q[0])$. Let $W_{0}=\left\{w_{m}^{0}\right\}$.

Suppose $w_{m}^{0} \neq u_{0}$ and $w_{m}^{0} \neq v_{0}$. Note $\left|F_{0}\right|=0$. By Lemma 3 , there are two disjoint paths $R_{1}=\left(u_{0}, v_{0}\right)$ and $R_{2}=$ $\left(s_{m}^{0}, w_{m}^{0}\right)$ covering $Q[0]-F_{0}$. Let $P_{j}=\left(P_{j}^{\prime}-s_{m}\right) \cup$ $\left\{u u_{0}, v v_{0}\right\} \cup R_{1}, \quad P_{i}=P_{i}^{\prime}(i \in\{1, \ldots, m-1\} \backslash\{j\}) \quad$ and $P_{m}=\left\{s_{m} s_{m}^{0}, w_{m}^{2} w_{m}^{0}\right\} \cup R_{2} \cup M_{m}$.

Suppose $w_{m}^{0}=u_{0}$. Let $z$ be the before vertex of $u$ in $P_{j}^{\prime}$ and $z_{0}=N(z) \cap V(Q[0])$. Note $\left|F_{0} \cup\left\{u_{0}\right\}\right|=1 \leq 2 n-$ 5 for $n \geq 3$. By Lemma 2 , there is a path $R=\left(z_{0}, v_{0}\right)$ covering $Q[0]-F_{0}-u_{0}$. Let $P_{j}=P_{j}^{\prime}\left[s_{j}, z\right] \cup$ $\left\{z z_{0}, v v_{0}\right\} \cup R \cup P_{j}^{\prime}\left[v, t_{j}\right], P_{i}=P_{i}^{\prime}(i \in\{1, \ldots, m-1\} \backslash\{j\})$ and $P_{m}=\left\{s_{m} u, u u_{0}, w_{m}^{0} w_{m}^{2}\right\} \cup M_{m}$. Similar to $w_{m}^{0}=v_{0}$.

Case 2.1.2.2. $\left|F_{0}\right|=1$.

Then, $\left|F_{i}\right| \leq 2 n-2 m-1$ for $i=1,2$. Since $\left|S_{1}\right|=$ $m>1=\left|F_{0}\right|$, there is a vertex $s_{i} \in S_{1}$ such that $s_{i}^{0} \notin F_{0}$ for $1 \leq i \leq m$. Without loss of generality, let $s_{m}^{0} \notin F_{0}$. Let $W_{1}=\left\{w_{i}^{1}: p+1 \leq i \leq m-1\right\}$ for $p \leq m-2, W_{1}=\varnothing$ for $p=m-1$. Then, $\left|W_{1}\right|=m-p-1$. Note $\left|T_{1}\right|+\left|W_{1}\right|=$ $p+(m-p-1)=m-1=\left|S_{1} \backslash\left\{s_{m}\right\}\right|$ and $\left|F_{1} \cup\left\{s_{m}\right\}\right| \leq$ $2 n-2 m$. By the induction hypothesis, there are $(m-1)$ disjoint paths $L_{i}=\left(s_{i}, t_{i}\right)(1 \leq i \leq p)$ and $L_{i}=\left(s_{i}\right.$, $\left.w_{i}^{1}\right)(p+1 \leq i \leq m-1)$ covering $Q[1]-F_{1}-s_{m}$. Let $P_{i}=L_{i}(1 \leq i \leq p)$ and $P_{i}=L_{i} \cup\left\{w_{i}^{1} w_{i}^{2}\right\} \cup M_{i}$ for $(p+$ $1 \leq i \leq m-1)$.

Let $W_{0}=\left\{w_{m}^{0}\right\}$. Note $\left|F_{0}\right|=1 \leq 2 n-5$ for $n \geq 3$. By Lemma 2, there is a path $R=\left(s_{m}^{0}, w_{m}^{0}\right)$ covering $Q[0]-F_{0}$. Let $P_{m}=\left\{s_{m} s_{m}^{0}\right\} \cup R \cup\left\{w_{m}^{0} w_{m}^{2}\right\} \cup M_{m}$.

Case 2.1.2.3. $\left|F_{0}\right| \geq 2$.

Then, $\left|F_{i}\right| \leq 2 n-2 m-2$ for $i=1,2$. Let $N_{Q[1]}\left(s_{m}\right)=$ $\left\{u_{1}, \ldots, u_{2 n-2}\right\}$ and $u_{i}^{0}=N\left(u_{i}\right) \cap V(Q[0])$ for $1 \leq$ $i \leq 2 n-2$. Since $\left|N_{Q[1]}\left(s_{m}\right)\right|-\left|S \backslash\left\{s_{m}\right\}\right|=2 n-2-(m-$ 1) $>2 n-2 m+1 \geq\left|F \cup\left\{w_{m}^{0}\right\}\right|$ for $m \geq 3$, there is an edge $u_{i} u_{i}^{0}(1 \leq i \leq 2 n-2)$ such that $u_{i} \in V\left(Q[1]-F_{1}-S_{1}\right)$ and $u_{i}^{0} \in V\left(Q[0]-F_{0}-\left\{w_{m}^{0}\right\}\right)$. Without loss of generality, let $u_{1} u_{1}^{0}$ be satisfied.

Let $W_{1}=\left\{w_{i}^{1}: p+1 \leq i \leq m-1\right\}$ for $p \leq m-2, W_{1}=\varnothing$ for $p=m-1$. Then, $\left|W_{1}\right|=m-p-1$. Note $\left|T_{1}\right|+\left|W_{1}\right|=p+(m-p-1)=m-1=\left|S_{1} \backslash\left\{s_{m}\right\}\right|$ and $\left|F_{1} \cup\left\{s_{m}, u_{1}\right\}\right| \leq 2 n-2 m$. By the induction hypothesis, there are $(m-1)$ disjoint paths $L_{i}=\left(s_{i}, t_{i}\right)(1 \leq i \leq p)$ and $L_{i}=\left(s_{i}, w_{i}^{1}\right)(p+1 \leq i \leq m-1)$ covering $Q[1]-$ $F_{1}-s_{m}-u_{1}$. Let $P_{i}=L_{i}(1 \leq i \leq p)$ and $P_{i}=L_{i} \cup\left\{w_{i}^{1}\right.$ $\left.w_{i}^{2}\right\} \cup M_{i}(p+1 \leq i \leq m-1)$.

Let $W_{0}=\left\{w_{m}^{0}\right\}$. Note $\left|F_{0}\right| \leq 2 n-2 m<2 n-5$ for $m \geq 3$. By Lemma 2 , there is a path $R=\left(u_{1}^{0}, w_{m}^{0}\right)$ covering $Q[0]-F_{0}$. Let $P_{m}=\left\{s_{m} u_{1}, u_{1} u_{1}^{0}\right\} \cup R \cup\left\{w_{m}^{0} w_{m}^{2}\right\} \cup M_{m}$.

Case 2.1.3. $1 \leq\left|S_{1}\right| \leq m-1$.

Let $\left|S_{1}\right|=p$. Then, $\left|S_{2}\right|=m-p$. Let $S_{1}=\left\{s_{1}, \ldots, s_{p}\right\}$ and $S_{2}=\left\{s_{p+1}, \ldots, s_{m}\right\}$. If $\left|T_{1}\right|=m$ or $\left|T_{2}\right|=m$, then it is similar to Case 2.1.2. Thus, $1 \leq\left|T_{i}\right| \leq m-1$ for $i=1,2$. Let $1 \leq\left|T_{1}\right|=q<p=\left|S_{1}\right| \leq m-1$. Then, $1 \leq\left|T_{2}\right|=$ $m-q \leq m-1$. Let $\quad T_{1}=\left\{t_{1}, \ldots, t_{q}\right\} \quad$ and $T_{2}=\left\{t_{q+1}, \ldots, t_{m}\right\}$.

Let $W_{j}=\left\{w_{i}^{j}: q+1 \leq i \leq p-1\right\}$ for $p-q \geq 2$ and $W_{j}=$ $\varnothing$ for $p-q=1$ where $j=1,2$. Then, $\left|W_{1}\right|=\left|W_{2}\right|=p-q-1 \leq m-3$. Since $|V(Q[i])|-$ $|F|-|S|-|T|-\left|W_{i}\right| \geq 3^{n-1}-(2 n-2 m)-2 m-(m-$ $3) \geq 3$ for $3 \leq m \leq n$, there are two edges $u_{0} u_{1}, v_{0} v_{2}$ such that $\left\{u_{i}, v_{i}\right\} \subset V\left(Q[i]-F-S-T-W_{i}\right)$ for $0 \leq i \leq 2$. Note $\left|F_{0}\right|=2 n-2 m<2 n-5$ for $m \geq 3$. By Lemma 2, there is a path $R=\left(u_{0}, v_{0}\right)$ covering $Q[0]-F_{0}$.

Note $\quad\left|W_{2}\right|+\left|\left\{v_{2}\right\}\right|+\left|S_{2}\right|=(p-q-1)+1+(m-$ $p)=m-q=\left|T_{2}\right|$. By the induction hypothesis, there are $(m-q)$ disjoint paths $M_{i}=\left(w_{i}^{2}, t_{i}\right)(q+$ $1 \leq i \leq p-1), M_{p}=\left(v_{2}, t_{p}\right)$ and $M_{i}=\left(s_{i}, t_{i}\right)(p+1 \leq$ $i \leq m)$ from $S_{2} \cup W_{2} \cup\left\{v_{2}\right\}$ to $T_{2}$ covering $Q[2]-F_{2}$.

Note $\quad\left|S_{1}\right|=p=q+(p-q-1)+1=\left|T_{1}\right|+\left|W_{1}\right|+$ $\left|\left\{u_{1}\right\}\right|$. By the induction hypothesis, there are $p$ disjoint paths $L_{i}=\left(s_{i}, t_{i}\right)(1 \leq i \leq q), \quad L_{i}=\left(s_{i}, w_{i}^{1}\right)(q+1 \leq$ $i \leq p-1), L_{p}=\left(s_{p}, u_{1}\right)$ from $S_{1}$ to $T_{1} \cup W_{1} \cup\left\{u_{1}\right\}$ covering $Q[1]-F_{1}$.

Let $P_{i}=L_{i}(1 \leq i \leq q), \quad P_{i}=L_{i} \cup\left\{w_{i}^{1} w_{i}^{2}\right\} \cup M_{i}(q+1 \leq$ $i \leq p-1), P_{p}=L_{p} \cup\left\{u_{1} u_{0}\right\} \cup R \cup\left\{v_{0} v_{2}\right\} \cup M_{p}$ and $P_{i}=$ $M_{i}(p+1 \leq i \leq m)$.

Case 2.2. $1 \leq\left|S_{0}\right|=\left|T_{0}\right| \leq m-1$.

By the induction hypothesis, there are $\left|S_{0}\right|$ disjoint paths from $S_{0}$ to $T_{0}$ covering $Q[0]-F_{0}$. Then, $1 \leq\left|S_{1}\right|-\left|T_{1}\right|=x=\left|T_{2}\right|-\left|S_{2}\right| \leq m-1$. Let $W_{j}=\left\{w_{i}^{j}\right.$ : $1 \leq i \leq x\}$ for $j=1,2$. Then, $\left|T_{1}\right|+\left|W_{1}\right|=\left|S_{1}\right| \leq m-1$ and $\left|S_{2}\right|+\left|W_{2}\right|=\left|T_{2}\right| \leq m-1$. By the induction hypothesis, there are $\left|S_{1}\right|$ (resp. $\left|T_{2}\right|$ ) disjoint paths from $S_{1}$ to $T_{1} \cup W_{1}$ (resp. $S_{2} \cup W_{2}$ to $T_{2}$ ) covering $Q[1]-F_{1}$ (resp. Q[2] $-F_{2}$ ).

Case 3. $\left|S_{i}\right| \neq\left|T_{i}\right|$ for all $0 \leq i \leq 2$. 
By Case 2, there are $m$ cycles of length three $C_{i}=$ $\left\langle w_{i}^{0}, w_{i}^{1}, w_{i}^{2}, w_{i}^{0}\right\rangle$ for $1 \leq i \leq m$ such that $w_{i}^{j} \in V(Q[j]-$ $\left.F_{j}-S-T\right)$ for $0 \leq j \leq 2$.

Case 3.1. $\left|S_{i}\right|>\left|T_{i}\right|$ for only one $0 \leq i \leq 2$.

Without loss of generality, let $\left|S_{0}\right|>\left|T_{0}\right|$. Then, $\left|S_{1}\right|<\left|T_{1}\right|, \quad\left|S_{2}\right|<\left|T_{2}\right|$. We get $\quad 1 \leq\left|S_{0}\right|-\left|T_{0}\right|=$ $\left(\left|T_{1}\right|-\left|S_{1}\right|\right)+\left(\left|T_{2}\right|-\left|S_{2}\right|\right) \leq m$.

Case 3.1.1. $\left|S_{0}\right|=m$ and $\left|T_{0}\right|=0$.

Then, $\left|S_{1}\right|=\left|S_{2}\right|=0$ and $1 \leq\left|T_{1}\right|,\left|T_{2}\right| \leq m-1$. Let $\left|T_{1}\right|=p$ and $\left|T_{2}\right|=m-p$. Let $S_{0}=\left\{s_{1}, \ldots, s_{m}\right\}$, $T_{1}=\left\{t_{1}, \ldots, t_{p}\right\}$ and $T_{2}=\left\{t_{p+1}, \ldots, t_{m}\right\}$. Without loss of generality, let $\left|T_{1}\right|=p \geq m-p=\left|T_{2}\right|$. Then, $2 p \geq m \geq 3$. We get $p \geq 2$ and $m-p \leq m-2$. Let $W_{2}=\left\{w_{i}^{1}: p+1 \leq i \leq m\right\}$. By the induction hypothesis, there are $(m-p)$ disjoint paths $M_{i}=\left(w_{i}^{2}\right.$, $\left.t_{i}\right)(p+1 \leq i \leq m)$ from $W_{2}$ to $T_{2}$ covering $Q[2]-F_{2}$.

Case 3.1.1.1. $\left|F_{0}\right|=2 n-2 m$.

Let $\quad S_{0}^{\prime}=S_{0} \backslash\left\{s_{1}\right\}$. Then, $\quad\left|S_{0}^{\prime}\right|=m-1$. Let $W_{0}=\left\{w_{i}^{0}: 2 \leq i \leq m\right\}$. Note $\left|W_{0}\right|=m-1=\left|S_{0}^{\prime}\right|$. By the induction hypothesis, there are $(m-1)$ disjoint paths $R_{i}=\left(s_{i}, w_{i}\right)(2 \leq i \leq m)$ from $S_{0}^{\prime}$ to $W_{0}$ covering $Q[0]-F_{0}$. Then, $s_{1} \in R_{i}$ for some $2 \leq i \leq m$. Without loss of generality, let $s_{1} \in R_{2}$. Let $u$ be the before vertex of $s_{1}$ in $R_{2}$ and $u_{1}=N(u) \cap V(Q[1])$.

Suppose $u_{1} \in T_{1}$. Without loss of generality, let $u_{1}=t_{1}$. Let $W_{1}=\left\{w_{i}^{1}: 2 \leq i \leq p\right\}$. Note $\left|W_{1}\right|=p-1=\left|T_{1} \backslash\left\{t_{1}\right\}\right|$ and $\left|F_{1} \cup\left\{t_{1}\right\}\right|=1 \leq 2(n-1)-2(m-2) \leq 2(n-1)-$ $2(p-1)$. By the induction hypothesis, there are $(p-1)$ disjoint paths $L_{i}=\left(w_{i}^{1}, t_{i}\right)(2 \leq i \leq p)$ covering $Q[1]-$ $F_{1}-t_{1}$. Let $P_{1}=R_{2}\left[s_{1}, w_{2}^{0}\right] \cup\left\{w_{2}^{0} w_{2}^{1}\right\} \cup L_{2}, P_{2}=R_{2}\left[s_{2}\right.$, $u] \cup\left\{u t_{1}\right\}, \quad P_{i}=R_{i} \cup\left\{w_{i}^{0} w_{i}^{1}\right\} \cup L_{i}(3 \leq i \leq p)$ and $P_{i}=$ $R_{i} \cup\left\{w_{i}^{0} w_{i}^{2}\right\} \cup M_{i}(p+1 \leq i \leq m)$.

Suppose $u_{1} \notin T_{1}$. Note $\left|W_{1} \cup\left\{u_{1}\right\}\right|=\left|T_{1}\right|=p$. By the induction hypothesis, there are $p$ disjoint paths $L_{1}=\left(u_{1}, t_{1}\right), L_{i}=\left(w_{i}^{1}, t_{i}\right)(2 \leq i \leq p)$ from $W_{1} \cup\left\{u_{1}\right\}$ to $T_{1} \quad$ covering $Q[1]-F_{1}$. Let $P_{1}=R_{2}\left[s_{1}, w_{2}^{0}\right] \cup$ $\left\{w_{2}^{0} w_{2}^{1}\right\} \cup L_{2}, \quad P_{2}=R_{2}\left[s_{2}, u\right] \cup\left\{u u_{1}\right\} \cup L_{1}, \quad P_{i}=R_{i} \cup$ $\left\{w_{i}^{0} w_{i}^{1}\right\} \cup L_{i}(3 \leq i \leq p) \quad$ and $\quad P_{i}=R_{i} \cup\left\{w_{i}^{0} w_{i}^{2}\right\} \cup M_{i}$ $(p+1 \leq i \leq m)$.

Case 3.1.1.2. $\left|F_{0}\right|=2 n-2 m-1$.

Then, $\left|F_{1} \cup F_{2}\right| \leq 1$. Since $\left|S_{0}\right|=m>1 \geq\left|F_{1}\right|$, there is a vertex $s_{i}$ such that $s_{i}^{1} \notin F_{1}$ for $1 \leq i \leq m$. Without loss of generality, let $s_{1}^{1} \notin F_{1}$. Let $W_{0}=\left\{w_{i}^{0}: 2 \leq i \leq m\right\}$. Note $\left|S_{0} \backslash\left\{s_{1}\right\}\right|=m-1=\left|W_{0}\right|$ and $\left|F_{0} \cup\left\{s_{1}\right\}\right|=2 n-2 m$. By the induction hypothesis, there are $(m-1)$ disjoint paths $R_{i}=\left(s_{i}, w_{i}\right)(2 \leq i \leq m)$ from $S_{0} \backslash\left\{s_{1}\right\}$ to $W_{0}$ covering $Q[0]-F_{0}-s_{1}$. Let $P_{i}=R_{i} \cup\left\{w_{i}^{0} w_{i}^{2}\right\} \cup$ $M_{i}(p+1 \leq i \leq m)$.

Let $W_{1}=\left\{w_{i}^{1}: 2 \leq i \leq p\right\}$. Note $\left|W_{1}\right|+\left|\left\{s_{1}^{1}\right\}\right|=p=\left|T_{1}\right|$. By the induction hypothesis, there are $p$ disjoint paths $L_{1}=\left(s_{1}^{1}, t_{1}\right), L_{i}=\left(w_{i}^{1}, t_{i}\right)(2 \leq i \leq p)$ from $\left\{s_{1}^{1}\right\} \cup W_{1}$ to $T_{1}$ covering $Q[1]-F_{1}$. Let $P_{1}=\left\{s_{1} s_{1}^{1}\right\} \cup L_{1}$ and $P_{i}=R_{i} \cup\left\{w_{i}^{0} w_{i}^{1}\right\} \cup L_{i}(2 \leq i \leq p)$.

Case 3.1.1.3. $\left|F_{0}\right| \leq 2 n-2 m-2$.
If $s_{1}^{1} \notin F$, then it is similar to Case 3.1.1.2. Suppose $s_{1}^{1} \in F$. Let $N_{Q[0]}\left(s_{1}\right)=\left\{u_{1}, \ldots, u_{2 n-2}\right\}$ and $u_{i}^{1}=$ $N\left(u_{i}\right) \cap V(Q[1])$ for $1 \leq i \leq 2 n-2$. Since $\left|N_{Q[0]}\left(s_{1}\right)\right|-$ $\left|S \backslash\left\{s_{1}\right\}\right|=2 n-2-(m-1)>2 n-2 m-1+p \geq \mid F \backslash\left\{s_{1}^{1}\right\}$ $|+| T_{1} \mid$ for $m>p$, there is a vertex $u_{i} \in V\left(Q[0]-F_{0}-\right.$ $S)$ such that $u_{i}^{1} \in V\left(Q[1]-F_{1}-T_{1}\right)$. Without loss of generality, let $u_{1}, u_{1}^{1}$ be satisfied.

Let $W_{0}=\left\{w_{i}^{0}: 2 \leq i \leq m\right\}$ and $u_{1} \notin W_{0}$. Then, $\left|F_{0} \cup\left\{s_{1}, u_{1}\right\}\right| \leq 2 n-2 m$. By the induction hypothesis, there are $(m-1)$ disjoint paths $R_{i}=\left(s_{i}, w_{i}\right)(2 \leq i \leq m)$ from $S_{0} \backslash\left\{s_{1}\right\}$ to $W_{0}$ covering Q $[0]-F_{0}-s_{1}-u_{1}$. Let $P_{i}=R_{i} \cup\left\{w_{i}^{0} w_{i}^{2}\right\} \cup M_{i}(p+1 \leq i \leq m)$.

Let $W_{1}=\left\{w_{i}^{1}: 2 \leq i \leq p\right\}$. Note $\left|W_{1}\right|+\left|\left\{u_{1}^{1}\right\}\right|=p=\left|T_{1}\right|$. By the induction hypothesis, there are $p$ disjoint paths $L_{1}=\left(u_{1}^{1}, t_{1}\right), L_{i}=\left(w_{i}^{1}, t_{i}\right)(2 \leq i \leq p)$ from $\left\{u_{1}^{1}\right\} \cup W_{1}$ to $T_{1}$ covering Q[1] $-F_{1}$. Let $P_{1}=\left\{s_{1} u_{1}, u_{1} u_{1}^{1}\right\} \cup L_{1}$ and $P_{i}=R_{i} \cup\left\{w_{i}^{0} w_{i}^{1}\right\} \cup L_{i}(2 \leq i \leq p)$.

Case 3.1.2. $\left|S_{0}\right|=m$ and $\left|T_{0}\right| \geq 1$.

Let $S_{0}=\left\{s_{1}, \ldots, s_{m}\right\}$. Since $\left|S_{i}\right|<\left|T_{i}\right|$ for $i=1,2$, $1 \leq\left|T_{i}\right| \leq m-2$ for $i=0,1,2$. Let $1 \leq\left|T_{0}\right|=$ $m-p-q \leq m-2,1 \leq\left|T_{1}\right|=p \leq m-2$ and $1 \leq\left|T_{2}\right|=$ $q \leq m-2$. Let $T_{0}=\left\{t_{1}, \ldots, t_{m-p-q}\right\}, \quad T_{1}=\left\{t_{m-p-q+1}\right.$, $\left.\ldots, t_{m-q}\right\}$ and $T_{2}=\left\{t_{m-q+1}, \ldots, t_{m}\right\}$.

Case 3.1.2.1. $\left|F_{0}\right|=2 n-2 m$.

Then, $\left|F_{1}\right|=\left|F_{2}\right|=0$. Let $S_{1}^{\prime}=\left\{s_{i}^{1}: m-p-q+1 \leq\right.$ $i \leq m-q\}$ and $S_{2}^{\prime}=\left\{s_{i}^{2}: m-q+1 \leq i \leq m\right\}$. Note $\left|S_{1}^{\prime}\right|=$ $p=\left|T_{1}\right|$ and $\left|S_{2}^{\prime}\right|=q=\left|T_{2}\right|$. By the induction hypothesis, there are $p$ (resp. q) disjoint paths $L_{i}=\left(s_{i}^{1}, t_{i}\right)(m-p-q+1 \leq i \leq m-q) \quad\left(\right.$ resp. $\quad M_{i}=$ $\left.\left(s_{i}^{2}, t_{i}\right)(m-q+1 \leq i \leq m)\right)$ from $S_{1}^{\prime}$ to $T_{1}$ (resp. $S_{2}^{\prime}$ to $T_{2}$ covering $\mathrm{Q}[1]-F_{1}$ (resp. $\left.\mathrm{Q}[2]-F_{2}\right)$ ). Let $P_{i}=$ $\left\{s_{i} s_{i}^{1}\right\} \cup L_{i}(m-p-q+1 \leq i \leq m-q)$ and $P_{i}=\left\{s_{i} s_{i}^{2}\right\} \cup$ $M_{i}(m-q+1 \leq i \leq m)$.

Let $S_{0}^{\prime}=\left\{s_{i}: 1 \leq i \leq m-p-q\right\}$. Note $\left|S_{0}^{\prime}\right|=m-p-q=$ $\left|T_{0}\right|$ and $\left|F_{0}\right|+\left|S_{0}\right| S_{0}^{\prime} \mid=(2 n-2 m)+(p+q) \leq 2(n-$ $1)-2(m-p-q)$ for $p+q \geq 2$. By the induction hypothesis, there are $(m-p-q)$ disjoint paths $P_{i}=\left(s_{i}, t_{i}\right)(1 \leq i \leq m-p-q)$ from $S_{0}^{\prime}$ to $T_{0}$ covering $\mathrm{Q}[0]-F_{0}$.

Case 3.1.2.2. $\left|F_{0}\right|=2 n-2 m-1$.

Since $\left|F_{1} \cup F_{2}\right| \leq 1<m=\left|S_{0}\right|$, there is a vertex $s_{i}$ such that $s_{i}^{2} \notin F_{2}$ for $1 \leq i \leq m$. Without loss of generality, let $s_{m}^{2} \notin F_{2}$. Let $W_{0}=\left\{w_{i}^{0}: m-p-q+1 \leq i \leq m-1\right\}$ for $p+q \geq 2$. Note $\left|W_{0}\right|+\left|T_{0}\right|=(p+q-1)+(m-p-$ $q)=m-1=\left|S_{0} \backslash\left\{s_{m}\right\}\right|$ and $\left|F_{0} \cup\left\{s_{m}\right\}\right|=2 n-2 m$. By the induction hypothesis, there are $(m-1)$ disjoint paths $R_{i}=\left(s_{i}, t_{i}\right)(1 \leq i \leq m-p-q)$ and $R_{i}=$ $\left(s_{i}, w_{i}\right)(m-p-q+1 \leq i \leq m-1)$ from $S_{0}^{\prime} \backslash\left\{s_{m}\right\}$ to $T_{0} \cup W_{0}$ covering $Q[0]-F_{0}-s_{m}$. Let $P_{i}=R_{i}(1 \leq$ $i \leq m-p-q)$.

Let $W_{1}=\left\{w_{i}^{1}: m-p-q+1 \leq i \leq m-q\right\}$. Note $\left|T_{1}\right|=$ $\left|W_{1}\right|=p \leq m-2$ and $\left|F_{1}\right| \leq 1 \leq 2(n-1)-2(m-$ $2) \leq 2(n-1)-2 p$. By the induction hypothesis, there are $p$ disjoint paths $L_{i}=\left(w_{i}^{1}, t_{i}\right)(m-p-q+1 \leq$ 
$i \leq m-q)$ from $W_{1}$ to $T_{1}$ covering $Q[1]-F_{1}$. Let $P_{i}=R_{i} \cup\left\{w_{i}^{0} w_{i}^{1}\right\} \cup L_{i}(m-p-q+1 \leq i \leq m-q)$.

Let $W_{2}=\left\{w_{i}^{2}: m-q+1 \leq i \leq m-1\right\}$ for $q \geq 2$ and $W_{2}=\varnothing$ for $q=1$. Since $w_{i}^{2} \in V(Q[2]-F-S-T)$, $s_{m}^{2} \notin W_{2}$. Note $\left|T_{2}\right|=\left|W_{2} \cup\left\{s_{m}^{2}\right\}\right|=q \leq m-2$ and $\left|F_{2}\right| \leq 1 \leq 2(n-1)-2(m-2) \leq 2(n-1)-2 q$. By the induction hypothesis, there are $q$ disjoint paths $M_{i}=$ $\left(w_{i}^{2}, t_{i}\right)(m-q+1 \leq i \leq m-1)$ and $M_{m}=\left(s_{m}^{2}, t_{m}\right)$ from $W_{2} \cup\left\{s_{m}^{2}\right\}$ to $T_{2}$ covering $Q[2]-F_{2}$. Let $P_{i}=$ $R_{i} \cup\left\{w_{i}^{0} w_{i}^{2}\right\} \cup M_{i}(m-q+1 \leq i \leq m-1)$ and $P_{m}=$ $\left\{s_{m} s_{m}^{2}\right\} \cup M_{m}$.

Case 3.1.2.3. $\left|F_{0}\right| \leq 2 n-2 m-2$.

Let $N_{Q[0]}\left(s_{m}\right)=\left\{u_{1}, \ldots, u_{2 n-2}\right\}$ and $u_{i}^{2}=N\left(u_{i}\right) \cap$ $V(Q[2])$ for $1 \leq i \leq 2 n-2$. Since $\left|N_{Q[0]}\left(s_{m}\right)\right|-$ $\left|S \backslash\left\{s_{m}\right\}\right|=2 n-2-(m-1)>(2 n-2 m)+q \geq|F|+$

$\left|T_{2}\right|$ for $m-1>q$, there is a vertex $u_{i} \in V\left(Q[0]-F_{0}-\right.$ $\left.T_{0}\right)$ such that $u_{i}^{2} \in V\left(Q[2]-F_{2}-T_{2}\right)$. Without loss of generality, let $u_{1}$ be satisfied.

Let $W_{0}=\left\{w_{i}^{0}: m-p-q+1 \leq i \leq m-1\right\}$ and $u_{1} \notin W_{0}$. Note $\quad\left|S_{0}^{\prime}\right|=\left|S \backslash\left\{s_{m}\right\}\right|=m-1=\left|T_{0}\right|+\left|W_{0}\right| \quad$ and $\left|F_{0} \cup\left\{s_{m}, u_{1}\right\}\right|=2 n-2 m$. By the induction hypothesis, there are $(m-1)$ disjoint paths $R_{i}=\left(s_{i}, t_{i}\right)(1 \leq i \leq m-$ $p-q)$ and $R_{i}=\left(s_{i}, w_{i}\right)(m-p-q+1 \leq i \leq m-1)$ from $S_{0}^{\prime}$ to $T_{0} \cup W_{0}$ covering $Q[0]-F_{0}-s_{m}-u_{1}$. Let $P_{i}=R_{i}(1 \leq i \leq m-p-q)$.

Let $\quad W_{1}=\left\{w_{i}^{1}: m-p-q+1 \leq i \leq m-q\right\}$. Note $\left|T_{1}\right|=\left|W_{1}\right|=p \leq m-2$. By the induction hypothesis, there are $p$ disjoint paths $L_{i}=\left(w_{i}^{1}, t_{i}\right)(m-p-q+$ $1 \leq i \leq m-q)$ from $W_{1}$ to $T_{1}$ covering $Q[1]-F_{1}$. Let $P_{i}=R_{i} \cup\left\{w_{i}^{0} w_{i}^{1}\right\} \cup L_{i}(m-p-q+1 \leq i \leq m-q)$.

Let $W_{2}=\left\{w_{i}^{2}: m-q+1 \leq i \leq m-1\right\}$ for $q \geq 2$ and $W_{2}=\varnothing$ for $q=1$. Note $\left|T_{2}\right|=\left|W_{2} \cup\left\{u_{1}^{2}\right\}\right|=q \leq m-2$. By the induction hypothesis, there are $q$ disjoint paths $M_{i}=\left(w_{i}^{2}, t_{i}\right)(m-q+1 \leq i \leq m-1)$ and $M_{m}=\left(u_{1}^{2}, t_{m}\right)$ from $W_{2} \cup\left\{u_{1}^{2}\right\}$ to $T_{2}$ covering $Q[2]-F_{2}$. Let $P_{i}=R_{i} \cup\left\{w_{i}^{0} w_{i}^{2}\right\} \cup M_{i}(m-q+1 \leq i \leq m-1)$ and $P_{m}=$ $\left\{s_{m} u_{1}, u_{1} u_{1}^{2}\right\} \cup M_{m}$.

Case 3.1.3. $1 \leq\left|S_{0}\right| \leq m-1$.

If $\left|T_{i}\right|=m$ for some $1 \leq i \leq 2$, then it is similar to Case 3.1.1 or 3.1.2. Since $\left|T_{i}\right|>\left|S_{i}\right| \geq 0$ for $1 \leq i \leq 2$, $1 \leq\left|T_{i}\right| \leq m-1 \quad$ for $\quad 1 \leq i \leq 2$. Let $W_{0}=\left\{w_{i}^{0}\right.$ : $\left.1 \leq i \leq\left|S_{0}\right|-\left|T_{0}\right|\right\}, \quad W_{1}=\left\{w_{i}^{1}: 1 \leq i \leq\left|T_{1}\right|-\left|S_{1}\right|\right\} \quad$ and $W_{2}=\left\{w_{i}^{2}: 1 \leq i \leq\left|T_{2}\right|-\left|S_{2}\right|\right\}$. Thus, $\left|W_{0}\right|=\left|S_{0}\right|-\left|T_{0}\right|=$ $\left(\left|T_{1}\right|-\left|S_{1}\right|\right)+\left(\left|T_{2}\right|-\left|S_{2}\right|\right)=\left|W_{1}\right|+\left|W_{2}\right|$. Note $\left|S_{0}\right|=$ $\left|T_{0}\right|+\left|W_{0}\right| \leq m-1, \quad\left|T_{1}\right|=\left|S_{1}\right|+\left|W_{1}\right| \leq m-1 \quad$ and $\left|T_{2}\right|=\left|S_{2}\right|+\left|W_{2}\right| \leq m-1$. By the induction hypothesis, there are $\left|S_{0}\right|,\left|T_{1}\right|$ and $\left|T_{2}\right|$ disjoint paths from $S_{0}$ to $W_{0} \cup T_{0}$, from $S_{1} \cup W_{1}$ to $T_{1}$ and from $S_{2} \cup W_{2}$ to $T_{2}$ covering $Q[0]-F_{0}, \quad Q[1]-F_{1}$ and $Q[2]-F_{2}$, respectively.

Case 3.2. $\left|S_{i}\right|>\left|T_{i}\right|$ for two $0 \leq i \leq 2$.

There is only one $i$ such that $\left|S_{i}\right|<\left|T_{i}\right|$ for $0 \leq i \leq 2$. It is similar to Case 3.1.

\section{One-to-Many Disjoint Paths}

Lemma 4. Let $0 \leq i \leq 2$ be a 3-ary $n$-cube for $n \geq 2$. Let $F$ be a faulty set of $V\left(Q_{n}^{3}\right)$. Let $\left\{s, t_{1}, t_{2}\right\}$ be the subset of $V\left(Q_{n}^{3}-F\right)$. If $|F| \leq 2 n-3$, then there exist two disjoint paths from $s$ to $t_{i}(i=$ $1,2)$ covering $Q_{n}^{3}-F$.

Proof. Note $|F| \leq 2 n-3$ for $n \geq 2$. By Lemma 2, there is a path $P=\left(t_{1}, t_{2}\right)$ covering $Q_{n}^{3}-F$. Then, $s \in V(P)$. Thus, there are two disjoint paths $P^{-1}\left[s, t_{1}\right]$ and $P\left[s, t_{2}\right]$ covering $Q_{n}^{3}-F$.

Lemma 5. Let $Q_{n}^{3}$ be a 3-ary $n$-cube for $n \geq 3$. Let $F$ be a faulty set of $V\left(Q_{n}^{3}\right)$. Let $C=\left\langle s_{0}, s_{1}, s_{2}, s_{0}\right\rangle$ be a fault-free cycle of length three, $s \in V(C)$ and $T$ be the subset of $V\left(Q_{n}^{3}-F-\right.$ $V(C)$ ) with $|T|=3$. If $|F| \leq 2 n-4$, then there are three disjoint paths from $s$ to $T$ covering $Q_{n}^{3}-F$.

Proof. Let $T=\left\{t_{0}, t_{1}, t_{2}\right\}$. We decompose $Q_{n}^{3}$ into three isomorphic subgraphs $Q[0], Q[1], Q[2]$ along one dimension such that $s_{i} \in V(Q[i])$ for $i \in\{0,1,2\}$. Suppose there is $s t_{i} \in E\left(Q_{n}^{3}\right)$ for some $0 \leq i \leq 2$. Let $P_{i}=\left(s, t_{i}\right)$. Note $\left|F \cup\left\{t_{i}\right\}\right| \leq 2 n-3$. By Lemma 4 , there are two disjoint paths $P_{j}=\left(s, t_{j}\right)$ for $j \in\{0,1,2\} \backslash\{i\}$. Thus, the lemma holds. So, suppose $s t_{i} \notin E\left(Q_{n}^{3}\right)$ for all $0 \leq i \leq 2$. Let $T_{i}=T \cap V(Q[i])$ and $F_{i}=F \cap V(Q[i])$ for $0 \leq i \leq 2$. Without loss of generality, let $\left|T_{0}\right|>0$ and $s=s_{0}$.

Case $1 .\left|T_{0}\right|=1,\left|T_{1}\right|=2$ and $\left|T_{2}\right|=0$.

Let $T_{0}=\left\{t_{0}\right\}$ and $T_{1}=\left\{t_{1}, t_{2}\right\}$.

Case 1.1. $\left|F_{i}\right| \leq 2 n-5$ for $0 \leq i \leq 2$.

By Lemma 2, there is a Hamilton path $L=\left(t_{1}, t_{2}\right)$ in $Q[1]-F_{1}$. Since $|V(L)|-2|F| \geq 3^{n-1}-2(2 n-4)>0$ for $n \geq 3$, there is an edge $x y \in R$ such that $x_{i}, y_{i} \in V\left(Q[i]-F_{i}\right) \quad$ for $i=0,2$ where $x_{i}=$ $N(x) \cap V(Q[i])$ and $y_{i}=N(y) \cap V(Q[i])$. By Lemma 2 , there is a Hamilton path $M=\left(s_{2}, y_{2}\right)$ in $Q[2]-F_{2}$. By Lemma 4, there are two disjoint paths $R_{1}=\left(s, t_{0}\right)$ and $R_{2}=\left(s, x_{0}\right)$ covering $Q[0]-F_{0}$. Let $P_{0}=R_{1}, P_{1}=$ $R_{2} \cup\left\{x_{0} x\right\} \cup L^{-1}\left[x, t_{1}\right]$ and $P_{2}=\left\{s s_{2}\right\} \cup M \cup\left\{y_{2} y\right\} \cup$ $L\left[y, t_{2}\right]$.

Case 1.2. $\left|F_{i}\right|=2 n-4$ for some $0 \leq i \leq 2$.

Case 1.2.1. $\left|F_{0}\right|=2 n-4$.

Let $f \in F_{0}$ and $F_{0}^{\prime}=F_{0} \backslash\{f\}$. By Lemma 4, there are two disjoint paths $R_{1}=\left(s, t_{0}\right)$ and $R_{2}=(s, f)$ covering $Q[0]-F_{0}$. Let $u$ be the before vertex of $f$ in $R_{2}$ and $u_{1}=N(u) \cap V(Q[1])$.

$u_{1}=t_{1}$ or $u_{1}=t_{2}$. Without loss of generality, let $u_{1}=t_{1}$. Note $\left|F_{1} \cup\left\{t_{1}\right\}\right|=1 \leq 2 n-5$ for $n \geq 3$. By Lemma 2, there is a Hamilton path $L=\left(s_{1}, t_{2}\right)$ in $Q[1]-F_{1}-t_{1}$. Let $x y \in L, x_{2}=N(x) \cap V(Q[2])$ and $y_{2}=N(y) \cap V(Q[2])$. By Lemma 2, there is a Hamilton path $M=\left(x_{2}, y_{2}\right)$ in $Q[2]-F_{2}$. Let $P_{0}=R_{1}, P_{1}=$ 
$R_{2}[s, u] \cup\left\{u t_{1}\right\} \quad$ and $\quad P_{2}=\left\{s s_{1}\right\} \cup(L-x y) \cup$ $\left\{x x_{2}, y y_{2}\right\} \cup M$.

$u_{1} \neq t_{1}$ and $u_{1} \neq t_{2}$. Let $t_{2}^{2}=N\left(t_{2}\right) \cap V(Q[2])$. Note $\left|F_{1} \cup\left\{t_{2}\right\}\right|=1 \leq 2 n-5$ and $\left|F_{2}\right|=0$. By Lemma 2 , there is a Hamilton path $L=\left(u_{1}, t_{1}\right)$ and $M=\left(s_{2}, t_{2}^{2}\right)$ in $Q[1]-F_{1}-t_{2}$ and $Q[2]-F_{2}$, respectively. Let $P_{0}=R_{1}$, $P_{1}=R_{2}[s, u] \cup\left\{u u_{1}\right\} \cup L$ and $P_{2}=\left\{s s_{2}\right\} \cup M \cup\left\{t_{2}^{2} t_{2}\right\}$.

Case 1.2.2. $\left|F_{1}\right|=2 n-4$.

Let $f \in F_{1}$ and $F_{1}^{\prime}=F_{1} \backslash\{f\}$. By Lemma 4, there are two disjoint paths $L_{1}=\left(f, t_{1}\right)$ and $L_{2}=\left(f, t_{2}\right)$ covering $Q[1]-F_{1}^{\prime}$. Let $z_{i}$ be the next vertex of $f$ in $L_{i}$ for $i=1,2$ and $z_{i}^{j}=N(u) \cap V(Q[j])$ for $j=0,2$. By Lemma 4 , there are two disjoint paths $R_{1}=\left(s, t_{0}\right)$ and $R_{2}=\left(s, z_{1}^{0}\right)$ covering $Q[0]-F_{0}$. By Lemma 2, there is a Hamilton path $M=\left(s_{2}, z_{2}^{2}\right)$ in $Q[2]-F_{2}$. Let $P_{0}=R_{1}$, $P_{1}=R_{2} \cup\left\{z_{1}^{0} z_{1}\right\} \cup L_{1}\left[z_{1}, t_{1}\right]$ and $P_{2}=\left\{s s_{2}\right\} \cup M \cup$ $\left\{z_{2}^{2} z_{2}\right\} \cup L_{2}\left[z_{2}, t_{2}\right]$.

Case 1.2.3. $\left|F_{2}\right|=2 n-4$.

By Lemma 2, there is a Hamilton path $R=\left(s, t_{0}\right)$ in $Q[0]-F_{0}$. Let $f \in F_{2}$ and $F_{2}^{\prime}=F_{2} \backslash\{f\}$. By Lemma 2, there is a Hamilton path $M=\left(s_{2}, f\right)$ in $Q[2]-F_{2}^{\prime}$. Let $u$ be the before vertex of $f$ in $M$ and $u_{1}=N(u) \cap V(Q[1])$.

$u_{1}=t_{1}$ or $u_{1}=t_{2}$. Without loss of generality, let $u_{1}=t_{1}$. Note $\left|F_{1} \cup\left\{t_{1}\right\}\right|=1 \leq 2 n-5$ for $n \geq 3$. By Lemma 2 , there is a Hamilton path $L=\left(s_{1}, t_{2}\right)$ in $Q[1]-F_{1}-t_{1}$. Let $P_{0}=R$, $P_{1}=\left\{s s_{2}\right\} \cup M\left[s_{2}, u\right] \cup\left\{u t_{1}\right\}$ and $P_{2}=\left\{s s_{1}\right\} \cup L$.

$u_{1} \neq t_{1}$ and $u_{1} \neq t_{2}$. Since $u \neq s_{2}, u_{1} \neq s_{1}$. By Lemma 3 , there are two disjoint paths $L_{1}=\left(u_{1}, t_{1}\right)$ and $L_{2}=\left(s_{1}, t_{2}\right) \quad$ covering $Q[1]-F_{1}$. Let $P_{0}=R$, $P_{1}=\left\{s s_{2}\right\} \cup M\left[s_{2}, u\right] \cup\left\{u u_{1}\right\} \cup L_{1}$ and $P_{2}=\left\{s s_{1}\right\} \cup L$.

Case 2. $\left|T_{0}\right|=1,\left|T_{1}\right|=1$ and $\left|T_{2}\right|=1$.

Let $T_{0}=\left\{t_{0}\right\}, T_{1}=\left\{t_{1}\right\}$ and $T_{2}=\left\{t_{2}\right\}$.

Case 2.1. $\left|F_{i}\right| \leq 2 n-5$ for $0 \leq i \leq 2$.

By Lemma 2, there is a Hamilton path $R=\left(s, t_{0}\right), L=$ $\left(s_{1}, t_{1}\right)$ and $M=\left(s_{2}, t_{2}\right)$ in $Q[0]-F_{0}, Q[1]-F_{1}$ and $Q[2]-F_{2}$, respectively. Let $P_{0}=R, P_{1}=\left\{s s_{1}\right\} \cup L$ and $P_{2}=\left\{s s_{2}\right\} \cup M$.

Case 2.2. $\left|F_{i}\right|=2 n-4$ for some $0 \leq i \leq 2$.

Case 2.2.1. $\left|F_{0}\right|=2 n-4$.

Then, $\left|F_{1}\right|=\left|F_{2}\right|=0$. Since $\left|F_{0}\right|=2 n-4 \geq 2$ for $n \geq 3$, let $f \in F_{0}$ and $F_{0}^{\prime}=F_{0} \backslash\{f\}$. Then, $\left|F_{0}^{\prime}\right|=2 n-5$. By Lemma 4 , there are two disjoint paths $P=\left(s_{0}, t_{0}\right)$ and $R=\left(s_{0}, f\right)$ covering $Q[0]-F_{0}$. Then, $|V(R)| \geq 2$.

Case 2.2.1.1. $|V(R)|=2$.

Then, $R=\left\langle s_{0}, f\right\rangle$. By Lemma 2, there is a Hamilton path $P_{i}^{\prime}=\left(s_{i}, t_{i}\right)$ in $Q[i]$ for $i=1,2$. Let $P_{0}=P, P_{1}=$ $\left\{s s_{1}\right\} \cup P_{1}^{\prime}$ and $P_{2}=\left\{s s_{2}\right\} \cup P_{2}^{\prime}$.

Case 2.2.1.2. $|V(R)| \geq 3$.

Let $u$ be the before vertex of $f$ in $R$. Let $u_{i}=N(u) \cap V(Q[i])$ for $i \in\{1,2\}$. By the definition of $Q_{n}^{3}, u_{i} \in V\left(Q[i]-s_{i}\right)$ for $i \in\{1,2\}$.

Case 2.2.1.2.1. $u_{1}=t_{1}$ or $u_{2}=t_{2}$.
Without loss of generality, let $u_{1}=t_{1}$. Since $|V(Q[i])|-$ $\left|F_{i}\right|-\left|\left\{s_{i}, t_{1}, t_{2}\right\}\right|=3^{n-1}-3>1$ for $i=1,2$, there is an edge $x_{1} x_{2}$ such that $x_{i} \in V\left(Q[i]-F_{i}-\left\{s_{i}, t_{1}, t_{2}\right\}\right)$ for $i=1,2$. Note $\left|F_{1} \cup\left\{t_{1}\right\}\right|=1 \leq 2 n-5$ for $n \geq 3$. By Lemma 2, there is a Hamilton path $L=\left(s_{1}, x_{1}\right)$ in $Q[1]-F_{1}-t_{1}$. Note $\left|F_{2}\right|=0$. By Lemma 2 , there is a Hamilton path $M=\left(x_{2}, t_{2}\right)$ in $Q$ [2]. Let $P_{0}=P$, $P_{1}=R[s, u] \cup\left\{u t_{1}\right\}$, and $P_{2}=\left\{s s_{1}\right\} \cup L \cup\left\{x_{1} x_{2}\right\} \cup M$.

Case 2.2.1.2.2. $u_{1} \neq t_{1}$ and $u_{2} \neq t_{2}$.

By Lemma 2, there is a Hamilton path $L=\left(u_{1}, t_{1}\right)$ and $M=\left(s_{2}, t_{2}\right)$ in $Q[1]$ and $Q[2]$, respectively. Let $P_{0}=P$, $P_{1}=R[s, u] \cup\left\{u u_{1}\right\} \cup L, P_{1}=\left\{s s_{2}\right\} \cup M$.

Case 2.2.2. $\left|F_{1}\right|=2 n-4$ or $\left|F_{2}\right|=2 n-4$.

Let $\left|F_{1}\right|=2 n-4$. Then, $\left|F_{0}\right|=\left|F_{2}\right|=0$. Note $\left|F_{1}\right|=2 n-4 \geq 2$ for $n \geq 3$. Let $f \in F_{1}$. Then, $\left|F_{1} \backslash\{f\}\right|=2 n-5$. By Lemma 4, there are two disjoint paths $L_{1}=\left(s_{1}, t_{1}\right)$ and $L_{2}=\left(s_{1}, f\right)$ covering $Q[1]-F_{1}-f$.

Case 2.2.2.1. $\left|V\left(L_{2}\right)\right|=2$.

Then, $L_{2}=\left\langle s_{1}, f\right\rangle$. By Lemma 2, there is a Hamilton path $R=\left(s, t_{0}\right)$ and $M=\left(s_{2}, t_{2}\right)$ in $Q[0]$ and $Q[2]$, respectively. Let $P_{0}=R, \quad P_{1}=\left\{s s_{1}\right\} \cup L_{1} \quad$ and $P_{2}=\left\{s s_{2}\right\} \cup M$.

Case 2.2.2.2. $\left|V\left(L_{2}\right)\right|=3$.

Let $L_{2}=\left\langle s_{1}, u, f\right\rangle$. Let $u_{2}=N(u) \cap V(Q[2])$. Let $w$ be the later vertex of $s_{1}$ in $L_{1}$ and $w_{i}=N(w) \cap V(Q[i])$ for $i=0,2$.

Suppose $u_{2}=t_{2}$. Note $\left|F_{2} \cup\left\{t_{2}\right\}\right|=1 \leq 2 n-5$ for $n \geq 3$. By Lemma 2, there is a Hamilton path $M=\left(s_{2}, w_{2}\right)$ in $Q[2]-F_{2}-t_{2}$. By Lemma 2, there is a Hamilton path $R=\left(s, t_{0}\right)$ in $Q[0]$. Let $P_{0}=R, \quad P_{1}=\left\{s s_{2}\right\} \cup$ $M \cup\left\{w_{2} w\right\} \cup L_{1}\left[w, t_{1}\right]$ and $P_{2}=\left\langle s, s_{1}, u, t_{2}\right\rangle$.

Suppose $u_{2} \neq t_{2}$. By Lemma 2, there is a Hamilton path $M=\left(u_{2}, t_{2}\right)$ in $Q[2]$. By the definition of $Q_{n}^{3}$, $w_{0} \in N(s)$. Since $s t_{i} \notin E\left(Q_{n}^{3}\right), w_{0} \neq t_{0}$. By Lemma 4 , there are two disjoint paths $R_{1}=\left(s, t_{0}\right)$ and $R_{2}=\left(s, w_{0}\right) \quad$ covering $Q[0]-F_{0}$. Let $P_{0}=R_{1}$, $P_{1}=R_{2} \cup\left\{w_{0} w\right\} \cup L_{1}\left[w, t_{1}\right]$ and $P_{2}=\left\langle s, s_{1}, u, u_{2}\right\rangle \cup M$.

Case 2.2.2.3. $\left|V\left(L_{2}\right)\right| \geq 4$.

By Lemma 2, there is a Hamilton path $R=\left(s, t_{0}\right)$ in $Q[0]$. Let $u$ and $v$ be the later and before vertex of $s_{1}$ and $f$ in $L_{2}$, respectively. Let $u_{2}=N(u) \cap V(Q[2])$ and $v_{2}=N(v) \cap V(Q[2])$.

Suppose $t_{2}=u_{2}$ or $t_{2}=v_{2}$. Without loss of generality, let $t_{2}=u_{2}$. Note $\left|F_{2} \cup\left\{t_{2}\right\}\right|=1 \leq 2 n-5$ for $n \geq 3$. By Lemma 2, there is a Hamilton path $M=\left(s_{2}, v_{2}\right)$ in $Q[2]-F_{2}-t_{2}$. Let $P_{0}=R, \quad P_{1}=\left\{s s_{1}\right\} \cup L_{1} \quad$ and $P_{2}=\left\{s s_{2}\right\} \cup M \cup\left\{v_{2} v\right\} \cup L_{2}^{-1}[v, u] \cup\left\{u t_{2}\right\}$.

Suppose $t_{2} \neq u_{2}$ and $t_{2} \neq v_{2}$. By Lemma 3, there are two disjoint paths $M_{1}=\left(s_{2}, u_{2}\right)$ and $M_{2}=\left(v_{2}, t_{2}\right)$ covering $Q[2]-F_{2}$. Let $P_{0}=R, \quad P_{1}=\left\{s s_{1}\right\} \cup L_{1} \quad$ and $P_{2}=\left\{s s_{2}\right\} \cup M_{1} \cup\left\{u_{2} u\right\} \cup L_{2}[u, v] \cup\left\{v v_{2}\right\} \cup M_{2}$.

Case 3. $\left|T_{0}\right|=2,\left|T_{1}\right|=1$ and $\left|T_{2}\right|=0$.

Let $T_{0}=\left\{t_{0}, t_{1}\right\}$ and $T_{1}=\left\{t_{2}\right\}$. 
Case 3.1. $\left|F_{i}\right| \leq 2 n-6$ for all $0 \leq i \leq 2$.

Since $|V(Q[i])|-|F|-|T|-|\{s\}| \geq 3^{n-1}-(2 n-4)-$ $3-1>1$ for $n \geq 3$, there is an edge $x_{0} x_{2}$ such that $x_{i} \in V\left(Q[i]-F_{i}-T_{i}-s_{i}\right)$ for $i=0,2$. By Lemma 2 , there are two disjoint paths $R_{1}=\left(s, t_{0}\right)$ and $R_{2}=\left(x_{0}, t_{1}\right)$ covering $Q[0]-F_{0}$. By Lemma 3 , there is a Hamilton path $L=\left(s_{1}, t_{2}\right)$ and $M=\left(s_{2}, x_{2}\right)$ in $Q[1]-$ $F_{1}$ and $Q[2]-F_{2}$, respectively. Let $P_{0}=R_{1}$, $P_{1}=\left\{s s_{2}\right\} \cup M \cup\left\{x_{2} x_{0}\right\} \cup R_{2}$ and $P_{2}=\left\{s s_{1}\right\} \cup L$.

Case 3.2. $\left|F_{i}\right|=2 n-5$ for some $0 \leq i \leq 2$.

We get $\left|F_{j}\right| \leq 1 \leq 2 n-5$ for $n \geq 3$ and $j \in\{0,1,2\} \backslash\{i\}$. By Lemma 4, there are two disjoint paths $R_{1}=\left(s, t_{0}\right)$ and $R_{2}=\left(s, t_{1}\right)$ covering $\mathrm{Q}[0]-F_{0}$. Let $P_{0}=R_{1}, P_{1}=R_{2}$.

Since $|V(Q[i])|-|F|-|T|-\left|\left\{s_{i}\right\}\right| \geq 3^{n-1}-(2 n-4)-$ $3-1>1$, there is an edge $x_{1} x_{2}$ such that $x_{i} \in V(Q[i]-$ $\left.F_{i}-T_{i}-s_{i}\right)$ for $i=1,2$. Note $\left|F_{i}\right| \leq 2 n-5$ for $i=1,2$. By Lemma 2, there is a Hamilton path $L=\left(x_{1}, t_{2}\right)$ and $M=\left(s_{2}, x_{2}\right)$ in $Q[1]-F_{1}$ and $Q[2]-F_{2}$, respectively. Let $P_{2}=\left\{s s_{2}\right\} \cup M \cup\left\{x_{2} x_{1}\right\} \cup L$.

Case 3.3. $\left|F_{i}\right|=2 n-4$ for some $0 \leq i \leq 2$.

Case 3.3.1. $\left|F_{0}\right|=2 n-4$.

Then, $\left|F_{1}\right|=\left|F_{2}\right|=0$. By Lemma 2 , there is a Hamilton path $L=\left(s_{1}, t_{2}\right)$ in $Q[1]$. Let $P_{2}=\left\{s s_{1}\right\} \cup L$. Note $\left|F_{0}\right|=$ $2 n-4 \geq 2$ for $n \geq 3$. Let $f \in F_{0}$. Then, $\left|F_{0}^{\prime}\right|=\left|F_{0} \backslash\{f\}\right|=2 n-5$. By Lemma 4, there are two disjoint paths $P=\left(s, t_{0}\right)$ and $R=\left(s, t_{2}\right)$ covering $Q[0]-F_{0}^{\prime}$. Without loss of generality, let $f \in V(R)$. Then, $|V(R)| \geq 3$.

Case 3.3.1.1. $s f \in E(R)$ and $f t_{1} \in E(R)$.

Then, $R=\left\langle s_{0}, f, t_{1}\right\rangle$. Let $t_{1}^{2}=N\left(t_{1}\right) \cap V(Q[2])$. Note that $\left|F_{2}\right|=0$. By Lemma 2, there is a Hamilton path $M=\left(s_{2}, t_{1}^{2}\right) \quad$ in $Q[2]$. Let $P_{0}=P$ and $P_{1}=\left\{s s_{2}\right\} \cup M \cup\left\{t_{1}^{2} t_{1}\right\}$.

Case 3.3.1.2. $s f \in E(R)$ and $f t_{1} \notin E(R)$.

Let $u$ be the later vertex of $f$ in $R$ and $u_{2}=N(u) \cap V(Q[2])$. Note that $\left|F_{2}\right|=0$. By Lemma 2, there is a Hamilton path $M=\left(s_{2}, u_{2}\right)$ in $Q$ [2]. Let $P_{0}=$ $P$ and $P_{1}=\left\{s s_{2}\right\} \cup M \cup\left\{u_{2} u\right\} \cup R\left[u, t_{1}\right]$.

Case 3.3.1.3. $s f \notin E(R)$ and $f t_{1} \in E(R)$.

Let $w$ and $u$ be the later and before vertex of $s_{0}$ and $f$ in $P$ and $R$, respectively. Let $u_{2}=N(u) \cap V(Q[2]), w_{2}=$ $N(w) \cap V(Q[2])$ and $t_{1}^{2}=N\left(t_{1}\right) \cap V(Q[2])$. By the definition of $Q_{n}^{3}, u_{2}, w_{2}, t_{1}^{2}$ are different in $V\left(Q[2]-s_{2}\right)$. Note that $\left|F_{2}\right|=0 \leq 2 n-6$ for $n \geq 3$. By Lemma 3 , there are two disjoint paths $M_{1}=\left(s_{2}, t_{1}^{2}\right)$ and $M_{2}=\left(u_{2}, w_{2}\right)$ covering $Q[2]-F_{2}$. Let $P_{0}=R[s, u] \cup\left\{u u_{2}\right\} \cup$ $M_{2} \cup\left\{w_{2} w\right\} \cup P\left[w, t_{0}\right]$ and $P_{1}=\left\{s s_{2}\right\} \cup M_{1} \cup\left\{t_{1}^{2} t_{1}\right\}$.

Case 3.3.1.4. $s f \notin E(R)$ and $f t_{2} \notin E(R)$.

Let $u$ and $v$ be the before and later vertex of $f$ in $R$, respectively. Let $w$ be the later vertex of $s_{0}$ in $P$. Let $u_{2}=N(u) \cap V(Q[2]), \quad v_{2}=N(v) \cap V(Q[2]) \quad$ and $w_{2}=N(w) \cap V(Q[2])$. By the definition of $Q_{n}^{3}$, $u_{2}, v_{2}, w_{2}$ are different in $V\left(Q[2]-s_{2}\right)$. Note that $\left|F_{2}\right|=$
$0 \leq 2 n-6$ for $n \geq 3$. By Lemma 3, there are two disjoint paths $M_{1}=\left(s_{2}, v_{2}\right)$ and $M_{2}=\left(u_{2}, w_{2}\right)$ covering $Q[2]-F_{2}$. Let $P_{0}=R[s, u] \cup\left\{u u_{2}\right\} \cup M_{2} \cup\left\{w_{2} w\right\} \cup$ $P\left[w, t_{0}\right]$ and $P_{1}=\left\{s s_{2}\right\} \cup M_{1} \cup\left\{v_{2} v\right\} \cup R\left[v, t_{1}\right]$.

Case 3.3.2. $\left|F_{1}\right|=2 n-4$.

Note $\left|F_{1}\right|=2 n-4 \geq 2$ for $n \geq 3$. Let $f \in F_{1}$. Then, $\left|F_{1}^{\prime}\right|=\left|F_{1} \backslash\{f\}\right|=2 n-5$. By Lemma 4, there are two disjoint paths $L_{1}=\left(s_{1}, t_{2}\right)$ and $L_{2}=\left(s_{1}, f\right)$ covering $Q[1]-F_{1}^{\prime}$. Let $P_{2}=\left\{s s_{1}\right\} \cup L_{1}$. Then, $\left|V\left(L_{2}\right)\right| \geq 2$.

Case 3.3.2.1. $\left|V\left(L_{2}\right)\right|=2$.

Then, $L_{2}=\left\langle s_{1}, f\right\rangle$. Let $t_{1}^{2}=N\left(t_{1}\right) \cap V(Q[2])$. Note $\left|F_{0} \cup\left\{t_{1}\right\}\right|=1 \leq 2 n-5$ for $n \geq 3$. By Lemma 2 , there is a Hamilton path $R=\left(s, t_{0}\right)$ in $Q[0]-F_{0}-t_{1}$. Note $\left|F_{2}\right|=0$. By Lemma 2, there is a Hamilton path $M=$ $\left(s_{2}, t_{1}^{2}\right)$ in $Q$ [2]. Let $P_{0}=R$ and $P_{1}=\left\{s s_{2}\right\} \cup M \cup\left\{t_{1}^{2} t_{1}\right\}$.

Case 3.3.2.2. $\left|V\left(L_{2}\right)\right|=3$.

Let $L_{2}=\left\langle s_{1}, u, f\right\rangle, u_{i}=N(u) \cap V(Q[i])$ for $i=0,2$. Then, $u_{i} \in V\left(Q[i]-s_{i}\right)$ for $i=0,2$.

Suppose $u_{0}=t_{0}$ or $u_{0}=t_{1}$. Without loss of generality, let $u_{0}=t_{1}$. Note $\left|F_{0} \cup\left\{t_{1}\right\}\right|=1 \leq 2 n-5$ for $n \geq 3$. By Lemma 2 , there is a Hamilton path $R=\left(s, t_{0}\right)$ in $Q[0]-F_{0}-t_{1}$. Note $\left|F_{2}\right|=0$. By Lemma 2, there is a Hamilton path $M=\left(s_{2}, u_{2}\right)$ in $Q[2]$. Let $P_{0}=R$ and $P_{1}=\left\{s s_{2}\right\} \cup M \cup\left\{u_{2} u, u t_{1}\right\}$.

Suppose $u_{0} \neq t_{0}$ and $u_{0} \neq t_{1}$. Note $\left|F_{0}\right|=0 \leq 2 n-6$ for $n \geq 3$. By Lemma 3 , there are two disjoint paths $R_{1}=$ $\left(s, t_{0}\right)$ and $R_{2}=\left(u_{0}, t_{1}\right)$ covering $Q[0]-F_{0}$. Note $\left|F_{2}\right|=0$. By Lemma 2, there is a Hamilton path $M=$ $\left(s_{2}, u_{2}\right)$ in $Q[2]$. Let $P_{0}=R_{1}$ and $P_{1}=\left\{s s_{2}\right\} \cup$ $M \cup\left\{u_{2} u, u u_{0}\right\} \cup R_{2}$.

Case 3.3.2.3. $\left|V\left(L_{2}\right)\right| \geq 4$.

Let $u$ and $v$ be the later and before vertex of $s_{1}$ and $f$ in $L_{2}$, respectively. Let $u_{2}=N(u) \cap V(Q[2])$ and $v_{0}=N(v) \cap V(Q[0])$. Note $\left|F_{2}\right|=0$. By Lemma 2, there is a Hamilton path $M=\left(s_{2}, u_{2}\right)$ in $Q[2]$.

Suppose $v_{0}=t_{0}$ or $v_{0}=t_{1}$. Without loss of generality, let $v_{0}=t_{1}$. Note $\left|F_{0} \cup\left\{t_{1}\right\}\right|=1 \leq 2 n-5$ for $n \geq 3$. By Lemma 3 , there is a Hamilton path $R=\left(s, t_{0}\right)$ in $Q[0]-F_{0}-t_{1}$. Let $P_{0}=R$ and $P_{1}=\left\{s s_{2}\right\} \cup M \cup$ $\left\{u_{2} u\right\} \cup L_{2}[u, v] \cup\left\{v t_{1}\right\}$.

Suppose $v_{0} \neq t_{0}$ and $v_{0} \neq t_{1}$. Note $\left|F_{0}\right|=0 \leq 2 n-6$ for $n \geq 3$. By Lemma 2 , there are two disjoint paths $R_{1}=$ $\left(s, t_{0}\right)$ and $R_{2}=\left(v_{0}, t_{1}\right)$ covering $Q[0]-F_{0}$. Let $P_{0}=R_{1}$ and $P_{1}=\left\{s s_{1}\right\} \cup M \cup\left\{u_{2} u\right\} \cup L_{2}[u, v] \cup\left\{v v_{0}\right\} \cup R_{2}$.

Case 3.3.3. $\left|F_{2}\right|=2 n-4$.

Note $\left|F_{1}\right|=0$. By Lemma 2 , there is a Hamilton path $L=\left(s_{1}, t_{2}\right)$ in $Q[1]$. Let $P_{2}=\left\{s s_{1}\right\} \cup L$. Note $\left|F_{2}\right|=2 n-$ $4 \geq 2$ for $n \geq 3$. Let $f \in F_{2}$. Then, $\left|F_{2}^{\prime}\right|=$ $\left|F_{2} \backslash\{f\}\right|=2 n-5$. By Lemma 2, there is a Hamilton path $M=\left(s_{2}, f\right)$ in $Q[2]-F_{2}^{\prime}$. Let $w$ be the before vertex of $f$ in $M$. Let $w_{0}=N(w) \cap V(Q[0])$. Then, $w_{0} \in V\left(Q[0]-s_{0}\right)$.

Case 3.3.3.1. $w_{0}=t_{0}$ or $w_{0}=t_{1}$. 
Without loss of generality, let $w_{0}=t_{1}$. Note $\left|F_{0} \cup\left\{t_{1}\right\}\right|=1 \leq 2 n-5$ for $n \geq 3$. By Lemma 2 , there is a Hamilton path $R=\left(s, t_{0}\right)$ in $Q[0]-F_{0}-t_{1}$. Let $P_{0}=R$ and $P_{1}=\left\{s_{2}\right\} \cup M\left[s_{2}, w\right] \cup\left\{w t_{1}\right\}$.

Case 3.3.3.2. $w_{0} \neq t_{0}$ and $w_{0} \neq t_{1}$.

Note $\left|F_{0}\right|=0 \leq 2 n-6$ for $n \geq 3$. By Lemma 3 , there are two disjoint paths $R_{1}=\left(s, t_{0}\right)$ and $R_{2}=\left(w_{0}, t_{1}\right)$ covering $Q[0]-F_{0}$. Let $P_{0}=R_{1}$ and $P_{1}=\left\{s s_{2}\right\} \cup M\left[s_{2}, w\right] \cup\left\{w w_{0}\right\} \cup R_{2}$.

Case $4 .\left|T_{0}\right|=3,\left|T_{1}\right|=0$ and $\left|T_{2}\right|=0$.

Let $\quad T_{0}=\left\{t_{0}, t_{1}, t_{2}\right\}$. Since $|V(Q[i])|-|F|-$ $|T|-|\{s\}| \geq 3^{n-1}-(2 n-4)-3-1>2$ for $n \geq 3$, there are two cycles of length three $C_{i}=\left\langle w_{i}^{0}, w_{i}^{1}, w_{i}^{2}, w_{i}^{0}\right\rangle$ such that $w_{i}^{j} \in V\left(Q[j]-F_{j}-T_{j}-s_{j}\right)$ for $0 \leq j \leq 2$ and $i \in\{1,2\}$.

Case 4.1. $\left|F_{i}\right| \leq 2 n-6$ for all $0 \leq i \leq 2$.

Let $t_{i}^{j}=N\left(t_{i}\right) \cap V(Q[j])$ for $0 \leq i \leq 2$ and $j \in\{1,2\}$. Suppose $\left|F_{i}\right| \leq 2$ for $i \in\{1,2\}$. There is $t_{i}^{1} \in V(Q[1]-$ $F_{1}$ ) for $0 \leq i \leq 2$. Let $t_{2}$ be satisfied. By Lemma 2 , there is a Hamilton path $L=\left(w_{1}^{1}, t_{2}^{1}\right)$ and $M=\left(s_{2}, w_{1}^{2}\right)$ in $Q[1]-F_{1}$ and $Q[2]-F_{2}$, respectively. Note $\left|F_{0} \cup\left\{t_{2}\right\}\right| \leq 2 n-5$. By Lemma 4, there are two disjoint paths $R_{1}=\left(s, t_{0}\right)$ and $R_{2}=\left(s, t_{1}\right)$ covering $Q[0]-F_{0}$. Let $\quad P_{0}=R_{1}, \quad P_{1}=R_{2} \quad$ and $\quad P_{2}=\left\{s s_{2}\right\} \cup M \cup$ $\left\{w_{1}^{2} w_{1}^{1}\right\} \cup L \cup\left\{t_{2}^{1} t_{2}\right\}$.

Suppose $\left|F_{i}\right| \geq 3$ for $i \in\{1,2\}$. Then, $\left|F_{0}\right| \leq$ $2 n-4-6=2 n-10$. By Theorem 1 , there are three disjoint paths $R_{0}=\left(s, t_{0}\right)$ and $R_{i}=\left(w_{i}^{0}, t_{i}\right)(i=1,2)$ covering $Q[0]-F_{0}$. By Lemma 2 , there is a Hamilton path $L=\left(s_{1}, w_{1}^{1}\right)$ and $M=\left(s_{2}, w_{2}^{2}\right)$ in $Q[1]-F_{1}$ and $Q[2]-F_{2}$, respectively. Let $P_{0}=R_{0}, \quad P_{1}=\left\{s s_{1}\right\} \cup$ $L \cup\left\{w_{1}^{1} w_{1}^{0}\right\} \cup R_{1}$ and $P_{2}=\left\{s s_{2}\right\} \cup M \cup\left\{w_{2}^{2} w_{2}^{0}\right\} \cup R_{2}$.

Case 4.2. $\left|F_{i}\right|=2 n-5$ for some $0 \leq i \leq 2$.

Case 4.2.1. $\left|F_{0}\right|=2 n-5$.

Then, $\left|F_{1} \cup F_{2}\right| \leq 1$. Without loss of generality, let $\left|F_{1}\right|=$ 0 and $\left|F_{2}\right| \leq 1$. By Lemma 4 , there are two disjoint paths $R_{0}=\left(s, t_{0}\right)$ and $R_{1}=\left(s, t_{1}\right)$ covering $Q[0]-F_{0}$. Then, $t_{2} \in R_{i}$ for some $0 \leq i \leq 1$. Without loss of generality, let $t_{2} \in R_{1}$. Then, $R_{1}=R_{1}\left[s, t_{2}\right] \cup R_{1}\left[t_{2}, t_{1}\right]$. Let $z$ and $u$ be the later vertex of $s$ and $t_{2}$ in $R_{0}$ and $R_{1}$, respectively. Let $u_{i}=N(u) \cap V(Q[i])$ and $z_{i}=N(z) \cap V(Q[i])$ for $1 \leq i \leq 2$.

Suppose $u_{2} \in F_{2}$. Then, $z_{2} \notin F_{2}$. By Lemma 2, there is a Hamilton path $L=\left(s_{1}, u_{1}\right)$ and $M=\left(s_{2}, z_{2}\right)$ in $Q[1]-$ $F_{1}$ and $Q[2]-F_{2}$, respectively. Let $P_{0}=\left\{s s_{2}\right\} \cup$ $M \cup\left\{z_{2} z\right\} \cup R_{0}\left[z, t_{0}\right], P_{1}=\left\{s s_{1}\right\} \cup L \cup\left\{u_{1} u\right\} \cup R_{1}\left[u, t_{1}\right]$ and $P_{2}=R_{0}\left[s, t_{2}\right]$.

Suppose $u_{2} \notin F_{2}$. By Lemma 2, there is a Hamilton path $L=\left(s_{1}, z_{1}\right)$ and $M=\left(s_{2}, u_{2}\right)$ in $Q[1]-F_{1}$ and $Q[2]-F_{2}$, respectively. Let $P_{0}=\left\{s s_{1}\right\} \cup L \cup$ $\left\{z_{1} z\right\} \cup R_{0}\left[z, t_{0}\right], \quad P_{1}=\left\{s s_{2}\right\} \cup M \cup\left\{u_{2} u\right\} \cup R_{1}\left[u, t_{1}\right]$ and $P_{2}=R_{0}\left[s, t_{2}\right]$.

Case 4.2.2. $\left|F_{1}\right|=2 n-5$ or $\left|F_{2}\right|=2 n-5$.

Let $\left|F_{1}\right|=2 n-5$. Then, $\left|F_{0} \cup F_{2}\right| \leq 1$.
Case 4.2.2.1. $\left|F_{0}\right|=0$.

Since $\left|F_{2}\right| \leq 1$, there is a vertex $t_{i}$ such that $t_{i}^{2} \notin F_{2}$ where $t_{i}^{2}=N\left(t_{i}\right) \cap V(Q[2])$ for $0 \leq i \leq 2$. Let $t_{2}$ be satisfied. By Lemma 2 , there is a Hamilton path $L=\left(s_{1}, w_{1}^{1}\right)$ and $M=\left(w_{1}^{2}, t_{2}^{2}\right)$ in $Q[1]-F_{1}$ and $Q[2]-F_{2}$, respectively. Note $\left|F_{0} \cup\left\{t_{2}\right\}\right|=1 \leq 2 n-5$. By Lemma 4 , there are two disjoint paths $R_{0}=\left(s, t_{0}\right)$ and $R_{1}=\left(s_{0}, t_{1}\right)$ covering $Q[0]-F_{0}$. Let $\quad P_{0}=R_{0}, \quad P_{1}=R_{1} \quad$ and $P_{2}=\{s s 1\} \cup L \cup\left\{w_{1}^{1} w_{1}^{2}\right\} \cup M \cup\left\{t_{2}^{2} t_{2}\right\}$.

Case 4.2.2.2. $\left|F_{2}\right|=0$.

Then, $\left|F_{0}\right| \leq 1 \leq 2 n-5$ for $n \geq 3$. By Lemma 4 , there are two disjoint paths $R_{1}=\left(s_{0}, t_{1}\right)$ and $R_{2}=\left(s_{0}, t_{2}\right)$ covering $\mathrm{Q}[0]-F_{0}$. Then, $t_{0} \in R_{i}$ for some $1 \leq i \leq 2$. Without loss of generality, let $t_{0} \in R_{1}$. Then, $R_{1}=R_{1}\left[s_{0}, t_{0}\right] \cup R_{1}\left[t_{0}, t_{1}\right]$. Let $u$ and $v$ be the later vertex of $t_{0}$ and $s_{0}$ in $R_{1}$ and $R_{2}$, respectively. Let $u_{2}=$ $N(u) \cap V(Q[2])$ and $v_{2}=N(v) \cap V(Q[2])$. Since $|V(Q[i])|-|F|-\left|\left\{s_{i}, u_{i}, v_{i}\right\}\right| \geq 3^{n-1}-(2 n-4)-3 \geq 1$ for $n \geq 3$, there is an edge $z_{1} z_{2}$ such that $z_{i} \in V(Q[i]-$ $\left.F-\left\{s_{i}, u_{2}, v_{2}\right\}\right)$ for $1 \leq i \leq 2$.

Note $\left|F_{1}\right|=2 n-5$. By Lemma 2, there is a Hamilton path $L=\left(s_{1}, z_{1}\right)$ in $Q[1]-F_{1}$. Note $\left|F_{2}\right|=0 \leq 2 n-6$ for $n \geq 3$. By Lemma 3, there are two disjoint paths $M_{1}=\left(z_{2}, u_{2}\right)$ and $M_{2}=\left(s_{2}, v_{2}\right)$ covering $Q[2]-F_{2}$. Let $P_{0}=R_{1}\left[s_{0}, t_{0}\right], \quad P_{1}=\left\{s s_{1}\right\} \cup L \cup\left\{z_{1} z_{2}\right\} \cup M_{1} \cup$ $\left\{u_{2} u\right\} \cup R_{1}\left[u, t_{1}\right]$ and $P_{2}=\left\{s s_{2}\right\} \cup M_{2} \cup\left\{v_{2} v\right\} \cup$ $R_{2}\left[v, t_{2}\right]$.

Case 4.3. $\left|F_{i}\right|=2 n-4$ for some $0 \leq i \leq 2$.

Case 4.3.1. $\left|F_{0}\right|=2 n-4$.

Then, $\left|F_{1}\right|=\left|F_{2}\right|=0$. Note $\left|F_{0}\right|=2 n-4 \geq 2$ for $n \geq 3$. Let $f_{1}, f_{2} \in F_{0}$. Let $F_{0}^{\prime}=F_{0} \backslash\left\{f_{1}, f_{2}\right\} \cup\left\{t_{2}\right\}$. Then, $\left|F_{0}^{\prime}\right|=2 n-5$. By Lemma 4 , there are two disjoint paths $R_{0}=\left(s, t_{0}\right)$ and $R_{1}=\left(s, t_{1}\right)$ covering $Q[0]-F_{0}^{\prime}$. Then, $f_{1}, f_{2} \in R_{i}$ for $0 \leq i \leq 1$. Let $f_{1}, f_{2} \in R_{1}$. Let $u$ and $v$ be the before and later vertex of $f_{1}, u_{i}=N(u) \cap V(Q[i])$ and $v_{i}=N(v) \cap V(Q[i])$ for $1 \leq i \leq 2$. Let $x$ and $y$ be the before and later vertex of $f_{2}, x_{i}=N(x) \cap V(Q[i])$ and $y_{i}=N(y) \cap V(Q[i])$ for $1 \leq i \leq 2$. Note $\left|F_{1}\right|=\left|F_{2}\right|=0$. By Lemma 2, there is a Hamilton path $L=\left(u_{1}, v_{1}\right)$ in $Q[1]-F_{1}$. Let $t_{2}^{2}=N\left(t_{2}\right) \cap V(Q[2])$. By Lemma 3, there are two disjoint paths $M_{1}=\left(x_{2}, y_{2}\right)$ and $M_{2}=$ $\left(s_{2}, t_{2}^{2}\right)$ covering $Q[2]-F_{2}$. Let $P_{0}=R_{0}, P_{1}=\left(R_{1}-\right.$ $\left.\left\{f_{1}, f_{2}\right\}\right) \cup L \cup M$ and $P_{2}=\left\{s s_{2}\right\} \cup M_{2} \cup\left\{t_{2}^{2} t_{2}\right\}$. Similarly, $f_{1} \in R_{0}$ and $f_{2} \in R_{1}$.

Case 4.3.2. $\left|F_{1}\right|=2 n-4$ or $\left|F_{2}\right|=2 n-4$.

Let $\left|F_{1}\right|=2 n-4$. Then, $\left|F_{0}\right|=\left|F_{2}\right|=0$. Note $\left|F_{1}\right|=2 n-4 \geq 2$ for $n \geq 3$. Let $f \in F_{1}$. Then, $\left|F_{1}^{\prime}\right|=\left|F_{1} \backslash\{f\}\right|=2 n-5$. By Lemma 2, there is a Hamilton path $L=\left(s_{1}, f\right)$ in $Q[1]-F_{1}$. Let $u$ be the before vertex of $f$ in $L$ and $u_{2}=N(u) \cap V(Q[2])$. Since $|T|=3<1$, there is a $t_{i}$ such that $t_{i}^{2} \neq u_{2}$ where $t_{i}^{2}=$ $N\left(t_{i}\right) \cap V(Q[2])$ for $0 \leq i \leq 2$. Let $t_{2}$ be satisfied. Note $\left|F_{0} \cup\left\{t_{2}\right\}\right|=1 \leq 2 n-5$. By Lemma 4, there are two disjoint paths $R_{0}=\left(s, t_{0}\right)$ and $R_{1}=\left(s, t_{1}\right)$ covering $Q[0]-F_{0}-t_{2}$. By Lemma 2, there is a Hamilton path 
$M=\left(u_{2}, t_{2}^{2}\right)$ in $Q[2]-F_{2}$. Let $P_{0}=R_{0}, P_{1}=R_{1}$ and $P_{2}=\left\{s s_{1}\right\} \cup(L-f) \cup\left\{u u_{2}\right\} \cup M \cup\left\{t_{2}^{2} t_{2}\right\}$.

Theorem 2. Let $Q_{n}^{3}$ be a 3-ary $n$-cube for $n \geq 2$. Let $F$ be a vertex faulty set of $Q_{n}^{3}$. Let $C$ be a fault-free cycle of length three. Let $s \in V(C)$ and $T \subset V\left(Q_{n}^{3}-F-V(C)\right)$ with $2 \leq|T|=m \leq n$. If $|F| \leq 2 n-m-1$, then there are $m$ disjoint paths from $s$ to $T$ covering $Q_{n}^{3}-F$.

Proof. We prove the theorem by induction on $m$. If $m=2$, then $|T|=2$ and $|F| \leq 2 n-3$. By Lemma 4 , there are two disjoint paths from $s$ to $T$ covering $Q_{n}^{3}-F$. If $m=3$, then $|T|=3$ and $|F| \leq 2 n-4$. By Lemma 5 , there are three disjoint paths from $s$ to $T$ covering $Q_{n}^{3}-F$.

Assume the theorem is true for $m-1$. Now we prove the theorem holds on $m$ for $4 \leq m \leq n$. Let $T=\left\{t_{1}, \ldots, t_{m}\right\}$. Suppose there is $s t_{i} \in E\left(Q_{n}^{3}\right)$ for some $1 \leq i \leq m$. Then, $\left|T \backslash\left\{t_{i}\right\}\right|=m-1$ and $\left|F \cup\left\{t_{i}\right\}\right| \leq 2 n-m=2 n-(m-1)-1$. By the induction hypothesis, there are $(m-1)$ disjoint paths $P_{j}=\left(s, t_{j}\right)(j \in\{1, \ldots, m\} \backslash\{i\})$ covering $Q_{n}^{3}-F-t_{i}$. Let $P_{i}=\left\langle s, t_{i}\right\rangle$. Thus, $P_{1}, \ldots, P_{m}$ are $m$ disjoint paths covering $Q_{n}^{3}-F$. So, we suppose $s t_{i} \notin E\left(Q_{n}^{3}\right)$ for all $1 \leq i \leq m$.

Let $C=\left\langle s_{0}, s_{1}, s_{2}, s_{0}\right\rangle$. So, we can decompose $Q_{n}^{3}$ into three subgraphs $Q[0], Q[1], Q[2]$ along one dimension such that $s_{i} \in V(Q[i])(0 \leq i \leq 2)$. Let $T_{i}=T \cap V(Q[i])$ and $F_{i}=$ $F \cap V(Q[i])$ for $0 \leq i \leq 2$. Without loss of generality, let $\left|T_{0}\right| \geq 1$ and $s=s_{0}$.

Case 1. $\left|T_{0}\right|=m$.

Let $T_{0}=\left\{t_{1}, \ldots, t_{m}\right\}$ and $t_{i}^{j}=N\left(t_{i}\right) \cap V(Q[j])$ for $1 \leq i \leq m$ and $j=1,2$. Then, $\left|T_{1}\right|=\left|T_{2}\right|=0$.

Case 1.1. $2 n-m-2 \leq\left|F_{0}\right| \leq 2 n-m-1$.

Then, $\left|F_{1} \cup F_{2}\right| \leq 1$. Without loss of generality, let $\left|F_{1}\right|=$ 0 and $\left|F_{2}\right| \leq 1$. Let $T_{0}^{\prime}=T_{0} \backslash\left\{t_{m-1}, t_{m}\right\}$. Then, $\left|T_{0}^{\prime}\right|=m-2$. Note $\left|F_{0}\right| \leq 2 n-m-1=2(n-1)-$ $(m-2)-1$. By the induction hypothesis, there are $(m-2)$ disjoint paths $R_{i}=\left(s, t_{i}\right)(1 \leq i \leq m-2)$ covering $Q[0]-F_{0}$. Then, $t_{m-1}, t_{m} \in \cup_{i=1}^{m-2} V\left(R_{i}-t_{i}-s\right)$.

Case 1.1.1. $t_{m-1}, t_{m} \in V\left(R_{i}\right)$ for some $1 \leq i \leq m-2$.

Without loss of generality, let $t_{m-1}, t_{m} \in V\left(R_{1}\right)$. Let $t_{m-1}$ is before $t_{m}$ in $R_{1}$. Then, $R_{1}=R_{1}[s$, $\left.t_{m-1}\right] \cup R_{1}\left[t_{m-1}, t_{m}\right] \cup R_{1}\left[t_{m}, t_{1}\right]$. Let $u$ and $v$ be the later vertex of $t_{m-1}$ and $t_{m}$ on $R_{1}$, respectively. Let $u_{i}=N(u) \cap V(Q[i]) v_{i}=N(v) \cap V(Q[i])$ for $i=1,2$. Since $\left|F_{2}\right| \leq 1, u_{2} \notin F_{2}$ or $v_{2} \notin F_{2}$.

Suppose $u_{2} \notin F_{2}$. Note $\left|F_{2}\right| \leq 1 \leq 2 n-5$ for $n \geq 3$. By Lemma 2 , there is a Hamilton path $M=\left(s_{2}, u_{2}\right)$ in $Q[2]-F_{2}$. Note $\left|F_{1}\right|=0$. By Lemma 2 , there is a Hamilton path $L=\left(s_{1}, v_{1}\right)$ in $Q[1]$. Let $P_{1}=$ $\left\{s s_{1}\right\} \cup L \cup\left\{v_{1} v\right\} \cup R_{1}\left[v, t_{1}\right], \quad P_{i}=R_{i}(2 \leq i \leq m-2)$, $P_{m-1}=R_{1}\left[s, t_{m-1}\right]$ and $P_{m}=\left\{s s_{2}\right\} \cup M \cup\left\{u_{2} u\right\} \cup R_{1}\left[u, t_{m}\right]$. Similar to $v_{2} \notin F_{2}$. Similarly, $t_{m}$ is before $t_{m-1}$ on $R_{1}$.

Case 1.1.2. $t_{m-1} \in V\left(R_{i}\right), t_{m} \in V\left(R_{j}\right)$ for $1 \leq i, j \leq m-2$ and $i \neq j$.
Without loss of generality, let $t_{m-1} \in V\left(R_{1}\right)$ and $t_{m} \in V\left(R_{2}\right)$. Then, $R_{1}=R_{1}\left[s, t_{m-1}\right] \cup R_{1}\left[t_{m-1}, t_{1}\right]$ and $R_{2}=R_{2}\left[s, t_{m}\right] \cup R_{2}\left[t_{m}, t_{2}\right]$. Let $u$ and $v$ be the later vertex of $t_{m-1}$ and $t_{m}$ on $R_{1}$ and $R_{2}$, respectively. Let $u_{i}=N(u) \cap V(Q[i])$ and $v_{i}=N(v) \cap V(Q[i])$ for $i=1,2$. Since $\left|F_{2}\right| \leq 1, u_{2} \notin F_{2}$ or $v_{2} \notin F_{2}$.

Suppose $u_{2} \notin F_{2}$. By Lemma 2, there is a Hamilton path $L=\left(s_{1}, v_{1}\right)$ and $M=\left(s_{2}, u_{2}\right)$ in $Q[1]-F_{1}$ and $Q[2]-F_{2}$, respectively. Let $P_{1}=\left\{s s_{2}\right\} \cup M \cup$ $\left\{u_{2} u\right\} \cup R_{1}\left[u, t_{1}\right], \quad P_{2}=\left\{s s_{1}\right\} \cup L \cup\left\{v_{1} v\right\} \cup R_{2}\left[v, t_{2}\right]$, $P_{i}=R_{i}(3 \leq i \leq m-2), \quad P_{m-1}=R_{1}\left[s, t_{m-1}\right] \quad$ and $P_{m}=R_{2}\left[s, t_{m}\right]$. Similar to $v_{2} \notin F_{2}$.

Case 1.2. $\left|F_{0}\right|=2 n-m-3$.

Then, $\left|F_{1} \cup F_{2}\right| \leq 2$. Without loss of generality, let $\left|F_{1}\right| \leq 1$. Since $\left|T_{0}\right|=m>1 \geq\left|F_{1}\right|$, there is a vertex $t_{i}$ such that $t_{i}^{1} \notin F_{1}$ for $1 \leq i \leq m$. Without loss of generality, let $t_{m}^{1} \notin F_{1}$. Note $\left|F_{0} \cup\left\{t_{m}\right\}\right|=2 n-m-$ $2=2(n-1)-(m-1)-1$. By the induction hypothesis, there are $(m-1)$ disjoint paths $P_{i}=$ $\left(s, t_{i}\right)(1 \leq i \leq m-1)$ covering $Q[0]-F_{0}-t_{m}$.

Since $|V(Q[i])|-|F|-\left|\left\{s_{i}, t_{m}^{i}\right\}\right| \geq 3^{n-1}-(2 n-m-1)-$ $2 \geq 5$ for $n \geq 3$, there is an edge $w_{1} w_{2}$ such that $w_{i} \in V\left(Q[i]-F-\left\{s_{i}, t_{m}^{i}\right\}\right) \quad$ for $\quad i=1,2$. Note $\left|F_{1} \cup F_{2}\right| \leq 2 \leq 2 n-5$ for $n \geq m \geq 4$. By Lemma 2 , there is a Hamilton path $R=\left(w_{1}, t_{m}^{1}\right)$ and $L=\left(s_{2}, w_{2}\right)$ in $Q[1]-F_{1}$ and $Q[2]-F_{2}$, respectively. Let $P_{m}=\left\{s s_{2}\right\} \cup L \cup\left\{w_{2} w_{1}\right\} \cup R \cup\left\{t_{m}^{1} t_{m}\right\}$.

Case 1.3. $\left|F_{0}\right| \leq 2 n-m-4$.

If $t_{m}^{1} \notin F_{1}$, then it is similar to Case 1.2. Suppose $t_{m}^{1} \in F_{1}$. Let $T_{0}^{\prime}=T \backslash\left\{t_{m}\right\}$. Let $\quad N_{Q[0]}\left(t_{m}\right)=$ $\left\{u_{1}, \ldots, u_{2 n-2}\right\}$. Since $\left|N_{Q[0]}\left(t_{m}\right)\right|-\left|T_{0}^{\prime}\right|=2 n-2-(m-$ 1) $=2 n-m-1>2 n-m-2 \geq\left|F \backslash\left\{t_{m}^{1}\right\}\right|$, there is a vertex $u_{i} \in V\left(Q[0]-F_{0}-T_{0}-s\right)$ such that $u_{i}^{j} \notin\left(F_{j} \backslash\left\{t_{m}^{j}\right\}\right)$ where $u_{i}^{j}=N\left(u_{i}\right) \cap V(Q[j])$ for $1 \leq j \leq 2$ and $1 \leq i \leq 2 n-2$. Without loss of generality, let $u_{1}$ be satisfied. Note $\left|F_{0}\right|+\left|\left\{t_{m}, u_{1}\right\}\right| \leq 2 n-m-2=$ $2(n-1)-(m-1)-1$. By the induction hypothesis, there are $(m-1)$ disjoint paths $P_{i}=\left(s, t_{i}\right)(1 \leq i \leq m-$ 1) covering $Q[0]-F_{0}-\left\{t_{m}, u_{1}\right\}$. Since $|V(Q[i])|-|F|-$ $\left|\left\{s_{i}, t_{m}^{i}, u_{1}^{i}\right\}\right| \geq 3^{n-1}-(2 n-m-1)-3>1$ for $4 \leq m \leq n$, there is an edge $x_{1} x_{2}$ such that $x_{i} \in V\left(Q[i]-F_{i}-\right.$ $\left.\left\{s_{i}, t_{m}^{i}, u_{1}^{i}\right\}\right)$ for $i=1,2$. Note $\left|F_{i}\right| \leq 2 n-5$ for $i=1,2$. By Lemma 2 , there is a Hamilton path $L=\left(x_{1}, u_{1}^{1}\right)$ and $M=\left(s_{2}, x_{2}\right)$ in $Q[1]-F_{1}$ and $Q[2]-F_{2}$, respectively. Let $P_{m}=\left\{s s_{2}\right\} \cup M \cup\left\{x_{2} x_{1}\right\} \cup L \cup\left\{u_{1}^{1} u_{1}, u_{1} t_{m}\right\}$.

Case 2. $\left|T_{0}\right|=m-1$.

Let $T_{0}=\left\{t_{1}, \ldots, t_{m-1}\right\}, T_{1}=\left\{t_{m}\right\}$ and $\left|T_{2}\right|=0$.

Case 2.1. $\left|F_{0}\right|=2 n-m-1$.

Then, $\left|F_{1}\right|=\left|F_{2}\right|=0$. Let $T_{0}^{\prime}=T_{0} \backslash\left\{t_{m-1}\right\}$. Then, $\left|T_{0}^{\prime}\right|=m-2$. Note $\left|F_{0}\right|=2 n-m-1=2(n-1)-$ $(m-2)-1$. By the induction hypothesis, there are $(m-2)$ disjoint paths $R_{i}=\left(s, t_{i}\right)(1 \leq i \leq m-2)$ covering $Q[0]-F_{0}$. Then, $t_{m-1} \in V\left(R_{i}-t_{i}-s\right)$ for some $1 \leq i \leq m-2$. Without loss of generality, let $t_{m-1} \in R_{1}$. Let $u$ be the later vertex of $t_{m}$ on $R_{1}$ and $u_{2}=N(u) \cap V(Q[2])$. By Lemma 2, there is a 
Hamilton path $L=\left(s_{1}, t_{m}\right)$ and $M=\left(s_{2}, u_{2}\right)$ in $Q$ [1] and $Q[2]$, respectively. Let $P_{1}=\left\{s s_{2}\right\} \cup$ $M \cup\left\{u_{2} u\right\} \cup R_{1}\left[u, t_{1}\right], \quad P_{i}=R_{i}(2 \leq i \leq m-2), \quad P_{m-1}=$ $R_{1}\left[s, t_{m-1}\right]$ and $P_{m}=\left\{s s_{1}\right\} \cup L$.

Case 2.2. $\left|F_{0}\right| \leq 2 n-m-2$.

Note $\left|F_{0}\right| \leq 2 n-m-2=2(n-1)-(m-1)-1$. By the induction hypothesis, there are $(m-1)$ disjoint paths $P_{i}=\left(s, t_{i}\right)(1 \leq i \leq m-1)$ covering $Q[0]-F_{0}$. Since $|V(Q[i])|-|F|-\left|\left\{s_{i}, t_{m}\right\}\right| \geq 3^{n-1}-(2 n-m-1)-2>1$ for $4 \leq m \leq n$, there is an edge $x_{1} x_{2}$ such that $x_{i} \in V\left(Q[i]-F_{i}-\left\{s_{i}, t_{m}\right\}\right)$ for $i=1,2$. Note $\left|F_{i}\right| \leq 2 n-$ 5 for $i=1,2$. By Lemma 2, there is a Hamilton path $L=\left(x_{1}, t_{m}\right)$ and $M=\left(s_{2}, x_{2}\right)$ in $Q[1]-F_{1}$ and $Q[2]-F_{2}$, respectively. Let $P_{m}=\left\{s s_{2}\right\} \cup M \cup\left\{x_{2}\right.$ $\left.x_{1}\right\} \cup L$.

Case 3. $1 \leq\left|T_{0}\right| \leq m-2$.

Let $\left|T_{0}\right|=p$. Then, $1 \leq p \leq m-2$ and $2 \leq \mid T_{1} \cup$ $T_{2} \mid=m-p \leq m-1$.

Case 3.1. $\left|T_{1}\right|=0$ or $\left|T_{2}\right|=0$.

Without loss of generality, let $\left|T_{1}\right|=0$. Then, $2 \leq\left|T_{2}\right|=m-p \leq m-1$. Let $T_{0}=\left\{t_{1}, \ldots, t_{p}\right\}$ and $T_{2}=\left\{t_{p+1}, \ldots, t_{m}\right\}$. Let $t_{i}^{j}=N\left(t_{i}\right) \cap V(Q[j])$ for $p+$ $1 \leq i \leq m$ and $j=0,1$. Since $|V(Q[i])|-|F|-$ $|T|-\left|\left\{s_{i}\right\}\right| \geq 3^{n-1}-(2 n-m-1)-m-1>m-1 \geq m-$ $p$, there are $(m-p)$ cycles of length three $C_{i}=\left\langle w_{i}^{0}, w_{i}^{1}, w_{i}^{2}, w_{i}^{0}\right\rangle$ such that $w_{i}^{j} \in V\left(Q[j]-F_{j}-\right.$ $T_{j}-s_{j}$ ) for $0 \leq j \leq 2$ and $p+1 \leq i \leq m$.

Case 3.1.1. $\left|T_{0}\right|>\left|T_{2}\right|$.

Since $\left|T_{0}\right|=p>m-p=\left|T_{2}\right|$, we get $m \leq 2 p-1$. Let $W_{2}=\left\{w_{i}^{2}: p+1 \leq i \leq m-1\right\}$ for $m-p \geq 2$. Note $\left|W_{2} \cup\left\{s_{2}\right\}\right|=m-p=\left|T_{2}\right|$ and $\left|F_{2}\right| \leq 2 n-m-1 \leq$ $2(n-1)-2(m-p)$ for $m \leq 2 p-1$. By Theorem 1 , there are $(m-p)$ disjoint paths $M_{i}=$ $\left(w_{i}^{2}, t_{i}\right)(p+1 \leq i \leq m-1)$ and $M_{m}=\left(s_{2}, t_{m}\right)$ from $W_{2} \cup\left\{s_{2}\right\}$ to $T_{2}$ covering $Q[2]-F_{2}$.

Let $W_{1}=\left\{w_{p+1}^{1}\right\}$. Note $\left|F_{1}\right| \leq 2 n-5$. By Lemma 2, there is a Hamilton path $L=\left(s_{1}, w_{p+1}^{1}\right)$ in $Q[1]-F_{1}$. Let $W_{0}=\left\{w_{i}^{0}: p+2 \leq i \leq m-1\right\}$ for $m-p \geq 3$ and $W_{0}=\varnothing$ for $m-p=2$. Then, $\left|W_{0}\right|=m-p-2$. Note $\left|T_{0}\right|+\left|W_{0}\right|=p+(m-p-2)=m-2 \quad$ and $\left|F_{0}\right| \leq 2 n-m-1=2(n-1)-(m-2)-1$. By the induction hypothesis, there are $(m-2)$ disjoint paths $R_{i}=\left(s, t_{i}\right)(1 \leq i \leq p) \quad$ and $R_{i}=\left(s, w_{i}^{0}\right)(p+2 \leq i \leq m-1)$ from $s$ to $T_{0} \cup W_{0}$ covering $Q[0]-F_{0}$.

Let $P_{i}=R_{i}(1 \leq i \leq p), P_{p+1}=\left\{s s_{1}\right\} \cup L \cup\left\{w_{p+1}^{1} w_{p+1}^{2}\right\} \cup$ $R_{p+1}, \quad P_{i}=R_{i} \cup\left\{w_{i}^{0} w_{i}^{2}\right\} \cup M_{i}(p+2 \leq i \leq m-1) \quad$ and $P_{m}=\left\{s s_{2}\right\} \cup M_{m}$.

Case 3.1.2. $\left|T_{0}\right|=\left|T_{2}\right|$.

Since $\left|T_{0}\right|=p=m-p=\left|T_{2}\right|$, we get $m=2 p \geq 4$. Thus, $m-p=p \geq 2$.

Case 3.1.2.1. $\left|F_{2}\right|=2 n-m-1$.
Let $T_{2}^{\prime}=T_{2} \backslash\left\{t_{p+1}\right\}$. Then, $\left|T_{2}^{\prime}\right|=p-1$. Note $\left|F_{1}\right|=0$. By Lemma 2, there is a Hamilton path $L=\left(s_{1}, t_{p+1}^{1}\right)$ in Q[1].

Let $W_{2}=\left\{w_{i}^{2}: p+2 \leq i \leq m-1\right\}$ for $m-p \geq 3$ and $W_{2}=\varnothing$ for $m-p=2$. Then, $\left|W_{2}\right|=m-p-2=$ $p-2$. Note $\left|W_{2} \cup\left\{s_{2}\right\}\right|=\left|T_{2}^{\prime}\right|=p-1 \quad$ and $\left|F_{2} \cup\left\{t_{p+1}\right\}\right|=2 n-m=2(n-1)-2(p-1) \quad$ for $m=2 p$. By Theorem 1 , there are $(p-1)$ disjoint paths $M_{i}=\left(w_{i}^{2}, t_{i}\right)(p+2 \leq i \leq m-1)$ and $M_{m}=\left(s_{2}, t_{m}\right)$ from $W_{2} \cup\left\{s_{2}\right\}$ to $T_{2}^{\prime}$ covering $Q[2]-F_{2}-t_{p+1}$.

Let $W_{0}=\left\{w_{i}^{0}: p+2 \leq i \leq m-1\right\}$ for $m-p \geq 3$ and $W_{0}=\varnothing$ for $m-p=2$. Then, $\left|W_{0}\right|=m-p-2$. Note $\quad\left|T_{0}\right|+\left|W_{0}\right|=p+(m-p-2)=m-2 \quad$ and $\left|F_{0}\right| \leq 2 n-m-1=2(n-1)-(m-2)-1$. By the induction hypothesis, there are $(m-2)$ disjoint paths $R_{i}=\left(s, t_{i}\right)(1 \leq i \leq p)$ and $R_{i}=\left(s, w_{i}^{0}\right)(p+2 \leq i \leq m-$ 1) from $s$ to $T_{0} \cup W_{0}$ covering $Q[0]-F_{0}$.

Let $P_{i}=R_{i}(1 \leq i \leq p), \quad P_{p+1}=\left\{s s_{1}\right\} \cup L \cup\left\{t_{p+1}^{1} t_{p+1}\right\}$, $P_{i}=R_{i} \cup\left\{w_{i}^{0} w_{i}^{2}\right\} \cup M_{i}(p+2 \leq i \leq m-1)$, and $P_{m}=\left\{s s_{2}\right\} \cup M_{m}$.

Case 3.1.2.2. $\left|F_{2}\right| \leq 2 n-m-2$.

Let $\quad W_{2}=\left\{w_{i}^{2}: p+1 \leq i \leq m-1\right\}$ Note $\left|\left\{s_{2}\right\} \cup W_{2}\right|=1+(m-p-1)=m-p=p=\left|T_{2}\right|$ and $\left|F_{2}\right| \leq 2 n-m-2=2(n-1)-2 p$ for $m=2 p$. By Theorem 1 , there are $p$ disjoint paths $M_{i}=\left(w_{i}^{2}, t_{i}\right)(p+$ $1 \leq i \leq m-1)$ and $M_{m}=\left(s_{2}, t_{m}\right)$ from $W_{2} \cup\left\{s_{2}\right\}$ to $T_{2}$ covering $Q[2]-F_{2}$.

Let $W_{1}=\left\{w_{p+1}^{1}\right\}$. Note $\left|F_{1}\right| \leq 2 n-5$. By Lemma 2, there is a Hamilton path $L=\left(s_{1}, w_{p+1}^{1}\right)$ in $Q[1]-F_{1}$. Let $W_{0}=\left\{w_{i}^{0}: p+2 \leq i \leq m-1\right\}$ for $m-p \geq 3$ and $W_{0}=\varnothing$ for $m-p=2$. Then, $\left|W_{0}\right|=m-p-2$. Note $\left|T_{0}\right|+\left|W_{0}\right|=p+(m-p-2)=m-2$ and $\left|F_{0}\right| \leq 2 n-$ $m-1=2(n-1)-(m-2)-1$. By the induction hypothesis, there are $(m-2)$ disjoint paths $R_{i}=\left(s, t_{i}\right)(1 \leq i \leq p)$ and $R_{i}=\left(s, w_{i}^{0}\right)(p+2 \leq i \leq m-$ 1) from $s$ to $T_{0} \cup W_{0}$ covering $Q[0]-F_{0}$.

Let $P_{i}=R_{i}(1 \leq i \leq p), P_{p+1}=\left\{s s_{1}\right\} \cup L \cup\left\{w_{p+1}^{1} w_{p+1}^{2}\right\} \cup$ $M_{p+1}, \quad P_{i}=R_{i} \cup\left\{w_{i}^{0} w_{i}^{2}\right\} \cup M_{i}(p+2 \leq i \leq m-1)$, and $P_{m}=\left\{s s_{2}\right\} \cup M_{m}$.

Case 3.1.3. $\left|T_{0}\right|<\left|T_{2}\right|$.

Since $\left|T_{0}\right|=p<m-p=\left|T_{2}\right|$, we get $m>2 p \geq 2$.

Case 3.1.3.1. $\left|F_{2}\right| \leq 2 n-2 m+2 p-2$.

Note $\left|F_{2}\right| \leq 2 n-2 m+2 p-2=2(n-1)-2(m-p)$. Let $W_{2}=\left\{w_{i}^{2}: p+1 \leq i \leq m-1\right\}$. Note $\mid\left\{s_{2}\right\} \cup$ $W_{2}|=1+(m-p-1)=m-p=| T_{2} \mid$. By Theorem 1, there are $(m-p)$ disjoint paths $M_{i}=\left(w_{i}^{2}, t_{i}\right)$ $(p+1 \leq i \leq m-1)$ and $M_{m}=\left(s_{2}, t_{m}\right)$ from $W_{2} \cup\left\{s_{2}\right\}$ to $T_{2}$ covering $Q[2]-F_{2}$.

Let $W_{1}=\left\{w_{p+1}^{1}\right\}$. Note $\left|F_{1}\right| \leq 2 n-m-1 \leq 2 n-5$ for $m \geq 4$. By Lemma 2, there is a Hamilton path $L=\left(s_{1}, w_{p+1}^{1}\right)$ in $Q[1]-F_{1}$.

Let $W_{0}=\left\{w_{i}^{0}: p+2 \leq i \leq m-1\right\}$ for $m-p \geq 3$ and $W_{0}=\varnothing$ for $m-p=2$. Then, $\left|W_{0}\right|=m-p-2$. Note 
$\left|T_{0}\right|+\left|W_{0}\right|=p+(m-p-2)=m-2$ and $\left|F_{0}\right| \leq 2 n-$ $m-1=2(n-1)-(m-2)-1$. By the induction hypothesis, there are $(m-2)$ disjoint paths $R_{i}=\left(s, t_{i}\right)(1 \leq i \leq p)$ and $R_{i}=\left(s, w_{i}^{0}\right)(p+2 \leq i \leq m-$ 1) from $s$ to $T_{0} \cup W_{0}$ covering $Q[0]-F_{0}$.

Let $P_{i}=R_{i}(1 \leq i \leq p), P_{p+1}=\left\{s s_{1}\right\} \cup L \cup\left\{w_{p+1}^{1} w_{p+1}^{2}\right\} \cup$ $M_{p+1}, \quad P_{i}=R_{i} \cup\left\{w_{i}^{0} w_{i}^{2}\right\} \cup M_{i}(p+2 \leq i \leq m-1)$, and $P_{m}=\left\{s s_{2}\right\} \cup M_{m}$.

Case $\quad 3.1 .3 .2 . \quad 2 n-2 m+k p-1 \leq\left|F_{2}\right| \leq 2 n-2 m+$ $(k+1) p-2$ for $2 p \leq k p \leq m$.

Then, $\left|F_{1}\right| \leq 2 n-m-1-(2 n-2 m+k p-1)=m-k p$. Since $\quad\left|T_{2}\right|-\left|F_{1}\right| \geq(m-p)-(m-k p)=(k-1) p$, there are $(k-1) p$ vertices $t_{i} \in T_{2}$ such that $t_{i}^{1} \notin F_{1}$. Without loss of generality, let $Z=\left\{t_{i}: p+1 \leq i \leq k p\right\}$ satisfy above requirement. Let $Z_{1}=\left\{t_{i}^{1}: p+1 \leq i \leq k p\right\}$. Then, $\left|Z_{1}\right|=|Z|=(k-1) p$. Let $T_{2}^{\prime}=T_{2} \backslash Z$. Let $W_{1}=$ $\left\{w_{i}^{1}: p+2 \leq i \leq k p\right\}$ for $(k-1) p \geq 2$ and $W_{1}=\varnothing$ for $(k-1) p=1$. Note $\left|F_{1}\right| \leq m-k p \leq 2(n-1)-2$ $(k-1) p$ for $2 \leq 2 p \leq k p \leq m \leq n$. By Theorem 1 , there are $(k-1) p$ disjoint paths $L_{p+1}=\left(s_{1}, t_{p+1}^{1}\right)$ and $L_{i}=$ $\left(w_{i}^{1}, t_{i}^{1}\right)(p+2 \leq i \leq k p)$ from $W_{1} \cup\left\{s_{1}\right\}$ to $Z_{1}$ covering $\mathrm{Q}[1]-F_{1}$.

Case 3.1.3.2.1. $2 p \leq k p<m$.

Let $W_{2}=\left\{w_{i}^{2}: k p+1 \leq i \leq m-1\right\}$ for $m-k p \geq 2$ and $W_{2}=\varnothing$ for $m-k p=1$. Note $\left|\left\{s_{2}\right\} \cup W_{2}\right|=m-k p=$ $\left|T_{2} \backslash Z\right|$ and $\left|F_{2} \cup Z\right| \leq(2 n-2 m+(k+1) p-2)+(k-$ 1) $p=2 n-2 m+2 k p-2=2(n-1)-2(m-k p)$. By Theorem 1 , there are $(m-k p)$ disjoint paths $M_{i}=$ $\left(w_{i}^{2}, t_{i}\right)(k p+1 \leq i \leq m-1)$ and $M_{m}=\left(s_{2}, t_{m}\right)$ from $W_{2} \cup\left\{s_{2}\right\}$ to $T_{2}^{\prime}$ covering $Q[2]-F_{2}-Z$.

Let $W_{0}=\left\{w_{i}^{0}: p+2 \leq i \leq m-1\right\}$ for $m-p \geq 3$ and $W_{0}=\varnothing$ for $m-p=2$. Then, $\left|W_{0}\right|=m-p-2$. Note $\left|T_{0}\right|+\left|W_{0}\right|=p+(m-p-2)=m-2$ and $\left|F_{0}\right| \leq 2 n-$ $m-1 \leq 2(n-1)-(m-2)-1$. By the induction hypothesis, there are $(m-2)$ disjoint paths $R_{i}=\left(s, t_{i}\right)(1 \leq i \leq p)$ and $R_{i}=\left(s, w_{i}^{0}\right)(p+2 \leq i \leq m-$ 1) from $s$ to $T_{0} \cup W_{0}$ covering $Q[0]-F_{0}$.

Let $P_{i}=R_{i}(1 \leq i \leq p), P_{p+1}=\left\{s s_{1}\right\} \cup L_{p+1} \cup\left\{t_{p+1}^{1} t_{p+1}\right\}$, $P_{i}=R_{i} \cup\left\{w_{i}^{0} w_{i}^{1}\right\} \cup L_{i} \cup\left\{t_{i}^{1} t_{i}\right\}(p+2 \leq i \leq k p), P_{i}=R_{i} \cup$ $\left\{w_{i}^{0} w_{i}^{2}\right\} \cup M_{i}(k p+1 \leq i \leq m-1)$, and $P_{m}=\left\{s s_{2}\right\} \cup M_{m}$. Case 3.1.3.2.2. $k p=m$.

$2 n-m-1=2 n-2 m+k p-1 \leq\left|F_{2}\right| \leq 2 n-2 m+(k$ $+1) p-2=2 n-m+p-2 \leq 2 n-m-1$. Thus, $\left|T_{0}\right|=$ $p=1, \quad\left|T_{2}\right|=m-p=m-1, \quad\left|F_{2}\right|=2 n-m-1$ and $\left|F_{0}\right|=\left|F_{1}\right|=0$. Let $T_{2}^{\prime}=T_{2} \backslash\left\{t_{m}\right\}$. Then, $\left|T_{2}^{\prime}\right|=m-2$. Note $\left|F_{2}\right|=2 n-m-1=2(n-1)-(m-2)-1$. By the induction hypothesis, there are $(m-2)$ disjoint paths $M_{i}=\left(s_{2}, t_{i}\right)(2 \leq i \leq m-1)$ from $s_{2}$ to $T_{2}^{\prime}$ covering $Q[2]-F_{2}$. Then, $t_{m} \in R_{i}$ for some $2 \leq i \leq m-1$. Without loss of generality, let $t_{m} \in R_{m-1}$. Let $u$ and $z_{i}$ be the next vertex of $t_{m}$ and $s_{2}$ in $R_{m-1}$ and $R_{i}$ for $2 \leq i \leq m-2$, respectively. Let $u_{1}=N(u) \cap V(Q[1])$ and $z_{i}^{0}=N\left(z_{i}\right) \cap V(Q[0])$. By the definition of $Q_{n}^{3}$, $z_{i}^{0} \in N(s)$. Since $\quad s t_{i} \notin E\left(Q_{n}^{3}\right), \quad z_{i}^{0} \notin T_{0}$. Let $Z_{0}=\left\{z_{i}^{0}: 2 \leq i \leq m-2\right\}$. By Lemma 2, there is a Hamilton path $L=\left(s_{1}, u_{1}\right)$ in $Q[1]$. Note
$\left|T_{0}\right|+\left|Z_{0}\right|=m-2$. By the induction hypothesis, there are $(m-2)$ disjoint paths $R_{1}=\left(s, t_{1}\right)$ and $R_{i}=\left(s, z_{i}^{0}\right)(2 \leq i \leq m-2)$ from $s$ to $T_{0} \cup Z_{0}$ covering $Q[0]-F_{0}$.

Let $P_{1}=R_{1}, P_{i}=R_{i} \cup\left\{z_{i}^{0} z_{i}\right\} \cup M_{i}\left[z_{i}, t_{i}\right](2 \leq i \leq m-2)$, $P_{m-1}=\left\{s s_{1}\right\} \cup L \cup\left\{u_{1} u\right\} \cup M_{m-1}\left[u, t_{m-1}\right] \quad$ and $P_{m}=\left\{s s_{2}\right\} \cup M_{m-1}\left[s_{2}, t_{m}\right]$.

Case 3.2. $\left|T_{1}\right|=\left|T_{2}\right|=1$.

Then, $\left|T_{0}\right|=m-2$. Let $T_{0}=\left\{t_{1}, \ldots, t_{m-2}\right\}, T_{1}=\left\{t_{m-1}\right\}$ and $T_{2}=\left\{t_{m}\right\}$. Note $\left|F_{i}\right| \leq 2 n-5$ for $1 \leq i \leq 2$. By Lemma 2, there is a Hamilton path $L=\left(s_{1}, t_{m-1}\right)$ and $M=\left(s_{2}, t_{m}\right)$ in $Q[1]-F_{1}$ and $Q[2]-F_{2}$, respectively. Note $\left|F_{0}\right| \leq 2 n-m-1=2(n-1)-(m-2)-1$. By the induction hypothesis, there are $(m-2)$ disjoint paths $R_{i}=\left(s, t_{i}\right)(1 \leq i \leq m-2)$ covering $Q[0]-F_{0}$.

Let $\quad P_{i}=R_{i}(1 \leq i \leq m-2), \quad P_{m-1}=\left\{s s_{1}\right\} \cup L \quad$ and $P_{m}=\left\{s s_{2}\right\} \cup M$.

Case 3.3. $\left|T_{i}\right| \geq 2$ for $i=1,2$.

Let $\left|T_{1}\right|=p \quad$ and $\quad\left|T_{2}\right|=q$. Since $\quad 1 \leq\left|T_{0}\right|=$ $m-p-q \leq m-4,4 \leq p+q \leq m-1$. Without loss of generality, let $\left|T_{1}\right|=p \geq q=\left|T_{2}\right|$. Then, $m-3$ $\geq p \geq q \geq 2$. Since $1 \leq m-p-q \leq m-2 q$, we get $2 q+1 \leq m$. Let $T_{0}=\left\{t_{1}, \ldots, t_{m-p-q}\right\}, T_{1}=\left\{t_{m-p-q+1}\right.$, $\left.\ldots, t_{m-q}\right\}$ and $T_{2}=\left\{t_{m-q+1}, \ldots, t_{m}\right\}$.

Since $|V(Q[i])|-|F|-\quad|T|-\left|\left\{s_{i}\right\}\right| \geq 3^{n-1}-(2 n-$ $m-1)-m-1 \geq m-3 \geq p+q-2$, there are $(p+q-2)$ cycles $C_{i}=\left\langle w_{i}^{0}, w_{i}^{1}, w_{i}^{2}, w_{i}^{0}\right\rangle$ where $w_{i}^{j} \in V(Q[j]-F-$ $\left.T-s_{j}\right)$ for $m-p-q+2 \leq i \leq m-1$ and $1 \leq j \leq 2$.

Let $W_{2}=\left\{w_{i}^{2}: m-q+1 \leq i \leq m-1\right\}$ for $q \geq 2$. Then, $\left|W_{2} \cup\left\{s_{2}\right\}\right|=q=\left|T_{2}\right|$. Note $\left|F_{2}\right| \leq 2 n-m-1 \leq 2(n-$ 1) $-2 q$ for $m \geq 2 q+1$ By Theorem 1 , there are $q$ disjoint paths $M_{i}=\left(w_{i}^{2}, t_{i}\right)(m-q+1 \leq i \leq m-1) \quad$ and $M_{m}=\left(s_{2}, t_{m}\right)$ from $W_{2} \cup\left\{s_{2}\right\}$ to $T_{2}$ covering $Q[2]-F_{2}$.

Case 3.3.1. $m \geq 2 p+1$.

Let $W_{1}=\left\{w_{i}^{1}: m-p-q+2 \leq i \leq m-q\right\}$ for $p \geq 2$. Then, $\left|W_{1} \cup\left\{s_{1}\right\}\right|=p=\left|T_{1}\right|$. Note $\left|F_{1}\right| \leq 2 n-m-1$ $\leq 2(n-1)-2 p$ for $m \geq 2 p+1$. By Theorem 1 , there are $p$ disjoint paths $L_{m-p-q+1}=\left(s_{1}, t_{m-p-q+1}\right)$ and $L_{i}=\left(w_{i}^{1}, t_{i}\right)(m-p-q+2 \leq i \leq m-q)$ from $\left\{s_{1}\right\} \cup W_{1}$ to $T_{1}$ covering $Q[1]-F_{1}$.

Let $\quad W_{0}=\left\{w_{i}^{0}: m-p-q+2 \leq i \leq m-1\right\}$. Then, $\left|W_{0}\right|=p+q-2 . \quad$ Note $\quad\left|T_{0}\right|+\left|W_{0}\right|=(m-p-q)+$ $(p+q-2)=m-2$ and $\left|F_{0}\right| \leq 2 n-m-1=2(n-$ $1)-(m-2)-1$. By the induction hypothesis, there are $(m-2)$ disjoint paths $R_{i}=\left(s, t_{i}\right)(1 \leq i \leq m-p-q)$ and $R_{i}=\left(s, w_{i}^{0}\right)(m-p-q+2 \leq i \leq m-1)$ from $s$ to $T_{0} \cup W_{0}$ covering $Q[0]-F_{0}$.

Let $\quad P_{i}=R_{i}(1 \leq i \leq m-p-q), \quad P_{m-p-q+1}=\left\{s s_{1}\right\} \cup$ $L_{m-p-q+1}, \quad P_{i}=R_{i} \cup\left\{w_{i}^{0} w_{i}^{1}\right\} \cup L_{i}(m-p-q+2 \leq i \leq$ $m-q), \quad P_{i}=R_{i} \cup\left\{w_{i}^{0} w_{i}^{2}\right\} \cup M_{i}(m-q+1 \leq i \leq m-1)$ and $P_{m}=\left\{s s_{2}\right\} \cup L_{m}$.

Case 3.3.2. $2 p \geq m$.

Case 3.3.2.1. $\left|F_{0}\right| \geq 2 p-m+1$. 
Note $\left|F_{1}\right| \leq 2 n-m-1-(2 p-m+1)=2 n-2 p-2=$ $2(n-1)-2 p$. Then, it is similar to Case 3.3.1.

Case 3.3.2.2. $2 p-m-(k+1) q+1 \leq\left|F_{0}\right| \leq 2 p-m-k q$ for $0 \leq k p \leq 2 p-m$.

Then, $\quad\left|F_{1}\right| \leq 2 n-m-1-(2 p-m-(k+1) q+1)=$ $2 n-2 p+(k+1) q-2$ and $\left|F_{0}\right|+\left|T_{0}\right| \leq(2 p-m-$ $k q)+(m-p-q)=p-(k+1) q<p=\left|T_{1}\right|$. Since $\left|T_{2}\right|-\left|T_{0}\right|-\left|F_{0}\right| \geq p-(p-(k+1) q)=(k+1) q$, there are $(k+1) q$ vertices $t_{i} \in T_{1}$ such that $t_{i}^{0} \notin\left(F_{0} \cup T_{0}\right)$. Let $t_{i}(m-p-q+1 \leq i \leq m-p+k q)$ satisfy above requirement. Let $Z=\left\{t_{i}: m-p-q+1 \leq i \leq m\right.$ $-p+k q\}$ and $Z_{0}=\left\{t_{i}^{0}: m-p-q+1 \leq i \leq m-p+k q\right\}$. Then, $|Z|=\left|Z_{0}\right|=(k+1) q$. Note $\left|T_{1} \backslash Z\right|=p-(k+1)$ $q \geq p-(2 p-m)-q=m-p-q \geq 1$ and $\left|F_{1}\right|+|Z| \leq$ $(2 n-2 p+(k+1) q-2)+(k+1) q=2 n-2 p+2(k+$ 1) $q-2=2(n-1)-2(p-(k+1) q)$.

Let $W_{1}=\left\{w_{i}^{1}: m-p+k q+2 \leq i \leq m-q\right\}$ for $(k+1)$ $q \leq 2 p-m+1 \leq p-2$. Then, $\left|W_{1}\right|=p-(k+1) q-1$. By Theorem 1, there are $(p-(k+1) q)$ disjoint paths $L_{m-p+k q+1}=\left(s_{1}, t_{m-p+k q+1}\right)$ and $L_{i}=\left(w_{i}^{1}, t_{i}\right)(m-p+$ $k q+2 \leq i \leq m-q)$ from $W_{1} \cup\left\{s_{1}\right\}$ to $T_{1} \backslash Z$ covering $Q[1]-F_{1}-Z$.

Let $W_{0}=\left\{w_{i}^{0}: m-p+k q+2 \leq i \leq m-1\right\}$ for $k q \leq$ $2 p-m \leq p-3$. Then, $\left|W_{0}\right|=p-k q-2$. Note $\left|T_{0}\right|+\left|Z_{0}\right|+\left|W_{0}\right|=(m-p-q)+(k+1) q+(p-k q$ $-2)=m-2$ and $\left|F_{0}\right| \leq 2 n-m-1$. By the induction hypothesis, there are $(m-2)$ disjoint paths $R_{i}=\left(s, t_{i}\right)(1 \leq i \leq m-p-q) \quad$ and $\quad R_{i}=\left(s, t_{i}^{0}\right)(m-$ $p-q+1 \leq i \leq m-p+k q), \quad R_{i}=\left(s, w_{i}^{0}\right)(m-p+k q$ $+2 \leq i \leq m-1)$ from $s$ to $T_{0} \cup Z_{0} \cup W_{0}$ covering $Q[0]-F_{0}$.

Let $P_{i}=R_{i}(1 \leq i \leq m-p-q), P_{i}=R_{i} \cup\left\{t_{i}^{0} t_{i}\right\}(m-p-$ $q+1 \leq i \leq m-p+k q), \quad P_{m-p+k q+1}=\left\{s s_{1}\right\} \cup L_{m-p+k q+1}$, $P_{i}=R_{i} \cup\left\{w_{i}^{0} w_{i}^{1}\right\} \cup L_{i}(m-p+k q+2 \leq i \leq m-q), P_{i}=$ $R_{i} \cup\left\{w_{i}^{0} w_{i}^{2}\right\} \cup M_{i}(m-q+1 \leq i \leq m-1) \quad$ and $P_{m}=\left\{s s_{2}\right\} \cup M_{m}$.

\section{Conclusions}

In this paper, we studied $m$ disjoint paths from $S$ to $T$ covering $Q_{n}^{3}-F$ with $|F| \leq 2 n-2 m$ for $2 \leq|S|=|T|=m \leq n$. Furthermore, we determined there are $m$ disjoint paths from $s$ to $T$ covering $Q_{n}^{3}-F$ with $|F| \leq 2 n-m-1$ where $2 \leq|T|=$ $m \leq n$ and $s$ is in a fault-free cycle of length three. In future research, scholars can continue to study vertex disjoint path problem of $Q_{n}^{k}$ or other graphs.

\section{Data Availability}

No data were used to support this study.

\section{Conflicts of Interest}

The authors declare that they have no conflicts of interest.

\section{Acknowledgments}

The authors would like to thank the National Natural Science Foundation of China (61772010) for supporting this study.

\section{References}

[1] Y.-K. Shih and S.-S. Kao, "One-to-One disjoint path covers on $k$-ary $n$-cubes," Theoretical Computer Science, vol. 412, pp. 4513-4530, 2011.

[2] X.-J. Li, B. Liu, M. Ma, and J.-M. Xu, "Many-to-many disjoint paths in hypercubes with faulty vertices," Discrete Applied Mathematics, vol. 217, pp. 229-242, 2017.

[3] X.-B. Chen, "Many-to-many disjoint paths in faulty hypercubes," Information Sciences, vol. 179, pp. 31110-33115, 2009.

[4] S. Zhang and S. Wang, "Many-to-many disjoint path covers in $k$-ary n-cubes," Theoretical Computer Science, vol. 491, pp. 103-118, 2013.

[5] Y. Xiang, "Lain Stewart, one-to-many node-disjoint paths in (n, k)-star graphs," Discrete Applied Mathematics, vol. 158, no. 1, pp. 62-70, 2010.

[6] L. Liptak, E. Cheng, J.-S. Kim, and S. W. Kim, "One-to-many node-disjoint paths of hyper-star networks," Discrete Applied Mathematics, vol. 160, pp. 2006-2014, 2012.

[7] L. You, J. Fan, Y. Han, and X. Jia, "one-to-one disjoint path covers on alternating group graphs," Theoretical Computer Science, vol. 562, pp. 146-164, 2015.

[8] J. A. Bondy and U. S. R. Murty, Graph Theory, Springer, New York, NY, USA, 2007.

[9] D. Wang, An Tong, M. Pan, K. Wang, and S. Qu, "Hamiltonian-like properties of $k$-ary $n$-cubes," in Proceedings of 6 th International Conference on Parallel and Distributed Computing, Applications and Technologies (PDCAT05), pp. 10021007, Dalian, China, December 2005.

[10] M.-C. Yang, J. Jimmy, M. Tan, and L.-H. Hsu, "Hamiltonian circuit and linear array embeddings in faulty $k$-ary $n$-cubes," Journal of Parallel and Distributed Computing, vol. 67, no. 4, pp. 362-368, 2007.

[11] W. She, "Two fault-free vertex-disjoint paths in 3-ary $n$-cube with faulty vertices," Journal of Jiamusi University (Natural Science Edition), vol. 31, no. 6, pp. 929-932, 2013. 